This is a draft version of the IJRR article:

Towards Dynamic Trot Gait Locomotion: Design, Control, and Experiments with Cheetah-cub, a Compliant Quadruped Robot, Alexander Sprowitz, Alexandre Tuleu, Massimo Vespignani, Mostafa Ajallooeian, Emilie Badri, Auke Jan ljspeert.

The final, definitive version of this paper will be published in International Journal of Robotics Research, Volume 32, Issue 8, July 2013, pp. 933 - 951. doi:10.1177/0278364913489205 by SAGE Publications Ltd, All rights reserved. (C)

[Alexander Sprowitz] 


\title{
Towards Dynamic Trot Gait Locomotion-Design, Control, and Experiments with Cheetah-cub, a Compliant Quadruped Robot
}

\author{
Alexander Spröwitz, Alexandre Tuleu, Massimo Vespignani, \\ Mostafa Ajallooeian, Emilie Badri, Auke Jan Ijspeert
}

\begin{abstract}
We present the design of a novel compliant quadruped robot: Cheetahcub, and a series of locomotion experiments with fast trotting gaits. The robot's leg configuration is based on a spring-loaded, pantograph mechanism with multiple segments. A dedicated open loop locomotion controller was derived and implemented. Experiments were run in simulation and in hardware on flat terrain and with a step-down, demonstrating the robot's self-stabilizing properties. The robot reached a running trot with short flight phases with a maximum Froude number of $\mathrm{FR}=1.30$, or 6.9 body lengths per second. Morphological parameters such as the leg design also played a role. By adding distal in-series elasticity, self-stability and maximum robot speed improved. Our robot has several advantages, especially when compared to larger and stiffer quadruped robot designs. 1) It is, to the best of our knowledge, the fastest of all quadruped robots below $30 \mathrm{~kg}$ (in terms of Froude number and body lengths per second). 2) It shows self-stabilizing behavior over a large range of speeds with open loop control. 3) It is lightweight, compact, electrically powered. 4) It is cheap, easy to reproduce, robust, and safe to handle. This makes it an excellent tool for research of multi-segment legs in quadruped robots.
\end{abstract}

\section{Introduction}

We present our findings from simulation and hardware experiments with a small legged, trotting, quadruped robot, Cheetah-cub, which achieves high-speed locomotion on flat terrain. Cheetah-cub speeds up to $1.42 \mathrm{~m} \mathrm{~s}^{-1}$. This corresponds to a Froude number of $\mathrm{FR}=1.30$, or $v=6.9$ body lengths per second. Table 1 indicates that in the field of quadruped legged robots applying non-rotatory legs, Cheetah-cub is currently the fastest robot of its kind. In particular it shows the highest Froude number for all trotting robots and highest Froude number for quadruped robots lighter than $30 \mathrm{~kg}$.

All presented results are based on joint-space and open loop locomotion patterns. This approach is based on the idea that relatively simple tasks, such as

*A. Spröwitz, A. Tuleu, M. Vespignani, M. Ajallooeian, E. Badri, and A. J. Ijspeert are with the Biorobotics Laboratory, Institute of Bioengineering, Ecole Polytechnique Federal de Lausanne (EPFL), Switzerland. Corresponding author: Alexander Spröwitz, Email: alexander.sproewitz@epfl.ch 


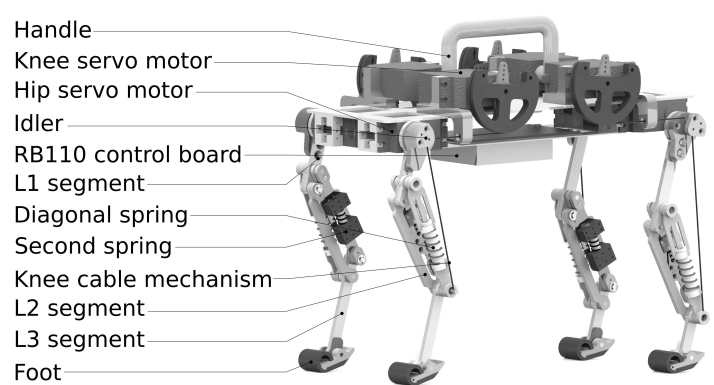

(a) Rendered robot, ASLP configuration

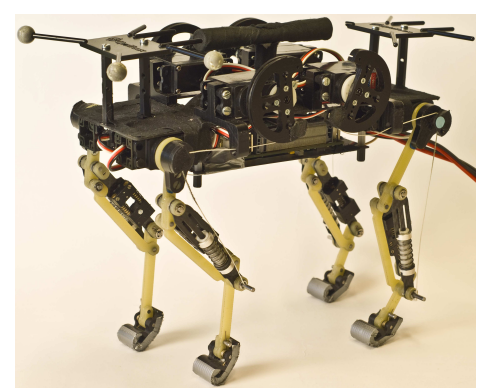

(b) ASLP configuration, hardware

Figure 1: Cheetah-cub robot in configuration ASLP. Distinguishing features are three-segment, pantograph, and passive compliant legs. An optional foot was mounted on a springy ankle joint (ASLP). The robot uses four RC servo motors at hip joints. Four RC servo motors are connected to the corresponding knee joints by a cable mechanism. They flex the robot's legs actively. A diagonal spring per leg extends the leg.

Table 1: (Literature review) Characteristics of quadruped robots, sorted by publication date. Bold-marked robots are energy autonomous. In case not provided in the corresponding publication, we calculated FR-values according to Alexander (1989), and estimated missing data. Maximum performance values per gait are marked with a grey color box. $\left.{ }^{1}\right]$ values are from personal communication with author. $\left[{ }^{2}\right] \mathrm{FR}$ values are estimated from the corresponding publication data.

\begin{tabular}{|c|c|c|c|c|c|c|c|}
\hline Robot (Author Year) & $\begin{array}{l}\mathrm{m}_{\text {rob }} \\
\mathrm{kg}\end{array}$ & $\begin{array}{l}\mathrm{h}_{\mathrm{hip}} \\
\mathrm{m}\end{array}$ & $\begin{array}{l}1_{\text {rob }} \\
\mathrm{m}\end{array}$ & $\begin{array}{l}v_{\max } \\
\mathrm{ms}^{-1}\end{array}$ & FR & $\begin{array}{l}\mathrm{BL} / \mathrm{s} \\
\mathrm{s}^{-1} \\
\end{array}$ & Gait \\
\hline \multirow[t]{2}{*}{ Quadruped (Raibert 1990) } & 38 & 0.56 & 0.78 & 2.2 & 0.88 & 2.8 & trot \\
\hline & 38 & 0.56 & 0.78 & 2.9 & 1.53 & 3.7 & bound \\
\hline Tekken $1\left[{ }^{2}\right]$ (Fukuoka et al. 2003) & 3.1 & 0.21 & 0.23 & 0.5 & 0.12 & 2.2 & walk \\
\hline Tekken1 $\left[{ }^{2}\right]$ (Fukuoka et al. 2009) & 3.1 & 0.21 & 0.23 & 1 & 0.49 & 4.3 & trot \\
\hline Tekken1 [2] (Fukuoka et al. 2009) & 3.1 & 0.21 & 0.23 & 1.1 & 0.59 & 4.8 & bound \\
\hline Aibo RES-210A $\left[^{2}\right]$ (Kohl et al. 2004) & 1.4 & 0.129 & 0.289 & 0.294 & 0.07 & 1 & walk \\
\hline Puppy $1[2]$ (Iida et al. 2004) & 1.5 & 0.2 & 0.17 & 0.5 & 0.13 & 2.9 & bound \\
\hline Scout II $\left[{ }^{2}\right]$ (Poulakakis et al. 2005) & 20.865 & 0.323 & 0.552 & 1.3 & 0.53 & 2.4 & bound \\
\hline Puppy II $\left.{ }^{2}\right]$ (Iida et al. 2005) & 0.273 & 0.075 & 0.142 & 0.5 & 0.34 & 3.5 & bound \\
\hline Tekken2 $\left[{ }^{2}\right]$ (Kimura et al. 2007) & 4.3 & 0.25 & 0.3 & 0.95 & 0.37 & 3.2 & trot \\
\hline \multirow[t]{3}{*}{ BigDog $\left[{ }^{2}\right]$ (Raibert et al. 2008) } & 109 & 1 & 1.1 & 3.1 & 0.98 & 2.8 & bound \\
\hline & 109 & 1 & 1.1 & 1.6 & 0.26 & 1.5 & trot \\
\hline & 109 & 1 & 1.1 & 2 & 0.41 & 1.8 & trot \\
\hline \multirow[t]{2}{*}{ KOLT $\left[{ }^{2}\right]$ (Estremera et al. 2008) } & 80 & 0.7 & 1.75 & 1.1 & 0.18 & 0.6 & trot \\
\hline & 80 & 0.7 & 1.75 & 1.06 & 0.16 & 0.6 & pronk \\
\hline Cheetah-2008 $\left[^{2}\right]$ (Rutishauser et al. 2008) & $\begin{array}{l}0.72 \\
0.72\end{array}$ & $\begin{array}{l}0.14 \\
0.14\end{array}$ & $\begin{array}{l}0.235 \\
0.235\end{array}$ & $\begin{array}{l}0.25 \\
0.11\end{array}$ & $\begin{array}{l}0.05 \\
0.01\end{array}$ & $\begin{array}{l}1.1 \\
0.5\end{array}$ & $\begin{array}{l}\text { walk } \\
\text { pace }\end{array}$ \\
\hline Rush [2] (Zhang et al. 2009) & 4.3 & 0.2 & 0.3 & 0.9 & 0.41 & 3 & bound \\
\hline PAW [ $\left.{ }^{2}\right]$ (Smith et al. 2010) & 15.7 & 0.212 & 0.494 & 1.2 & 0.69 & 2.4 & bound \\
\hline HyQ $\left[{ }^{1,2}, 2\right.$ (Semini et al. 2011) & 70 & 0.68 & 1 & 2.0 & 0.6 & 2.0 & trot \\
\hline Cheetah-cub & 1.1 & 0.158 & 0.205 & 1.42 & 1.30 & 6.9 & trot \\
\hline
\end{tabular}

rhythmic locomotion on flat terrain, should be performed almost "blindly", without the need for sensory feedback, or an explicit model of the robot. Quadruped robot locomotion could benefit from self-stabilizing properties of the compliant robot design. More complex locomotion tasks would easily be implemented on top of a simple open loop controller, and would then make use of sensory information. Our hope is that the design of higher level controllers for more complex locomotion tasks that require sensory information will be simplified by providing appropriate hardware, and robot-self-stabilizing open loop locomotion patterns. 
Here, we focused on identifying the main building blocks both at the level of hardware and control, to reach closer to what nature demonstrated already. The implementation of multi-segment, compliant legs presents a major biological solution to cover large distances, cross rough terrain, swim, climb trees, accelerate and decelerate swiftly, change directions, change gait and run energy efficiently, or jump. Yet, only a handful of quadruped robot platforms have demonstrated the ability for dynamic locomotion, which as a task represents a single example of what animals use their legs for. Legged robot platforms have been developed with continuously rotating legs, and show extremely fast locomotion speed, on rough terrain (Altendorfer et al. 2001; Schroer et al. 2004). Robots with continuously rotating legs apply a number of spoke-like mounted legs, attached to a fully rotating axis, similar to that of a wheel. Because of this mechanism, online changes of leg features are limited in such systems. In contrast, a robot with forward-rearward-forward swinging legs can for example apply asymmetric stance leg angles, or actively change its leg length during the stance phase, between touch-down and take-off.

We designed our robot with three-segment and four-segment front and hind legs. They are equipped with passive compliant elements, and are designed according to a pantograph leg configuration. Witte et al. (2001) proposed the pantograph leg template for robot leg designs in the parasagittal plane. The pantograph leg is based on findings of animal leg kinematics during cyclic locomotion, where proximal and distal leg segments keep their relative angular orientation during most parts of the locomotion cycle. The fixed angular relation deviates only during late stance phase, at the onset of toe-off.

When designing the robot hardware, we implemented and compared two pantograph structures: firstly, a three-segment, spring-loaded pantograph (SLP, Fig. 5) based on a regular four-bar mechanism, and secondly, a four-segment, advanced spring loaded pantograph (ASLP, Fig. 5) functionally more similar to the biological counterpart. Both leg implementations include a gravity-loaded leg spring. The hip actuator is mounted directly between the robot's body and leg. The knee actuator, acting in parallel to the main leg spring, is mounted proximally. The role of the knee actuator is only to retract the leg (by pulling on a cable) and not to extend the leg (leg extension is solely due to the springs). Our novel approach to robot leg design required an adapted approach to locomotion control. By considering principles from established locomotion controllers, we sought a stance phase leg length strategy, transitioning from low-speed to high-speed, while keeping the robot's body motion low (i.e. pitch and roll movements). The SLIP template (Blickhan 1989; Full et al. 1999) and Raibert's results from legged locomotion (Raibert et al. 1986) provide us with the insight that at higher robot speed, a robot's body momentum compresses passive, spring-loaded legs, depending on the robot's momentum, leg stiffness $k_{\text {leg }}$, and angle of attack between leg and ground. This momentum-triggered leg compression at higher-speed locomotion leads to a vertical mid-stance position of the hip joint below its touch-down height (running profile), and produces smooth vertical hip trajectory patterns. However, at lower speed the robot lacks momentum $(p=m \cdot v)$ to be converted into a sufficient spring deflecting force $(F=d p / d t)$. The choice of a proper leg stiffness is therefore critical. A leg of high stiffness will work well for high running speeds but not for low speeds. At low robot speed, a low momentum-based force will act on the springy leg $\left(k_{\text {leg }}^{\text {high }}\right)$, it will get the leg less compressed (Hooke's law), and the leg will remain at almost full 
length throughout the stance phase. The resulting higher pitch and roll body movements will destabilize the robot. On the contrary, a leg spring with lower stiffness $k_{\text {leg }}^{\text {low }}$ only works well at lower robot speeds. At higher robot speeds, the robot's legs would collapse. A solution to this problem is to combine highstiffness springs with actuation parallel to the leg spring. A spring-parallel force can be applied during the stance phase, acting on top of the momentum-based force in a higher stiffness leg $\left(k_{\text {leg }}^{\text {high }}\right)$. This parallel (additional) force helps compress the $k_{\text {leg }}^{\text {high }}$ legs in cases of low body-momentum, leading to lower pitch and roll body movements. At higher speed, its influence becomes less important as the leg springs are tuned for higher robot momentum. We implemented this as a cable mechanism (proximal knee actuator with cable mechanism) acting in the leg length direction, working in-parallel to above described momentumbased forces. The principle of a cable acting on a springy leg was shown earlier with the Bow-leg hopper (Zeglin 1999), for a different leg design, actuation, and leg control strategy. Further, the Cheetah-cub cable mechanism was carefully designed to work as a clutch, for cases where momentum-based forces exceeded cable-forces. This preserved the passive spring characteristics of the multi-segment leg. At high robot speed, the in-parallel knee actuation becomes unnecessary, and only momentum-based dynamics are required for leg length compression. During the swing phase, the knee-cable mechanism functions as a standard actuator for ground clearance, i.e. shortening the leg more than during the stance phase and avoiding that the forward swinging leg collides with the ground.

We propose two hypotheses, and tested them for their applicability. Firstly, we hypothesize that adding distal compliant elements to the $S L P$ leg design should increase robustness, speed, and improve cost of transport. The resulting $A S L P$ design is equipped with in-series elastic elements in form of a springy foot and an additional, Hamstring-like spring. In animals, distal, compliant segments are known to increase robustness, and to improve the cost of transport through subsequent energy storage and release (Alexander 1984a). Secondly, we hypothesize that a robot designed with passive elastic legs $\left(k_{l e g}^{h i g h}\right)$ and active leg shortening during stance phase can cover a large range of speeds on flat terrain, up to dynamic locomotion. We chose a Froude number FR $>1$ as an indicator for dynamic locomotion, i.e. a running trot with short flight phases. The remaining paper is organized as follows: in Section 2 we follow up on the state of the art of quadruped robot design, locomotion control, and extract bio-inspired hardware design principles. Section 3 describes the methods and materials we applied, including hardware and controller design, and experimental setup. We describe in detail our results from simulation and hardware experiments in Section 4. We discuss results and implications in Section 5, and conclude in Section 6.

\section{Related Work}

This section describes related work in the field of legged robotics, with focus on quadruped robots. The section further explains which type of research inspired the leg and controller design of the Cheetah-cub robot.

We focus on legged, quadruped robots with legs oscillating in a parasagittal (forward-backward and up-down) plane. This excludes very fast running legged 


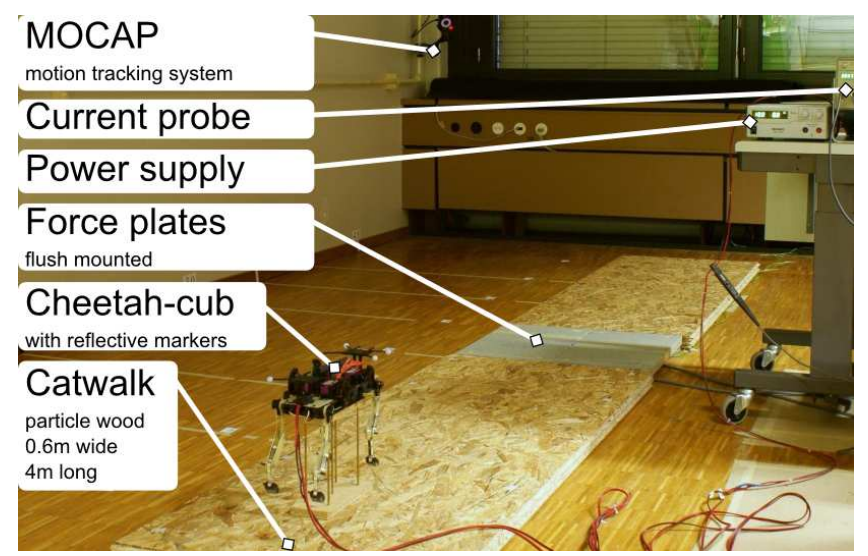

Figure 2: (Hardware experiment) The experimental setup was equipped with a high-speed camera mounted orthogonally to the cat-walk (not shown here). Twelve infrared motion capture cameras (MOCAP) extracted 6DOF data of the robot. A current probe and a digital oscilloscope were used for measuring the robot's power consumption. Two flush-mounted ground reaction force (GRF) plates provided left and right leg GRF data. The catwalk was roughly $4 \mathrm{~m}$ in length, and $0.6 \mathrm{~m}$ in width.

robots with continuously rotating legs (Altendorfer et al. 2001; Schroer et al. 2004), or legged but hexapedal robots like iSprawl (Kim et al. 2006). We describe legged, quadruped robots by their general leg design, degrees of freedom (DOF) per leg, and the type of actuation used.

Legged robot systems with a prismatic leg design (Fig. 4a) apply a linear sliding or telescopic mechanism, extending and shortening the leg along a line between foot and hip joint. Examples are Raibert's MIT Quadruped (Raibert et al. 1986) robot, and Scout II (Battaglia 1999). The MIT Quadruped robot actuates its legs by hydraulic pistons. It is able to actively protract, retract, adduct and abduct, as well as shorten and lengthen its legs. Scout II uses an electrical hip actuator and passive compliant prismatic legs. Both MIT Quadruped and Scout II, but also other similar quadruped robots showed versatile, dynamic, and fast gaits.

Typically two active DOF are implemented for active ground clearance. With the ability to actively shorten legs, the foot ground clearance can easily be provided independently from the body motion and orientation. However, already single hip DOF legged robots are capable of dynamical locomotion, as shown with PAW robot (Smith et al. 2010). These designs seek smart bodypitching control schemes, for example in combination with bounding gaits. Effectively, body-pitching at the right moment lifts up the hip or shoulder joints, and legs have sufficient ground clearance for a short moment of swing phase. However, gaits such as trot and walk require swing leg protraction that is independent from the body pitching movement. A single, active DOF presents a balanced solution between robot's weight, complexity, and gait versatility. Instead of a second active joint per leg, a lock-and-release mechanism can be implemented. It bends the robot's knees through passive leg dynamics, and allows for foot ground clearance (Hawker et al. 2000). Examples for single-active 


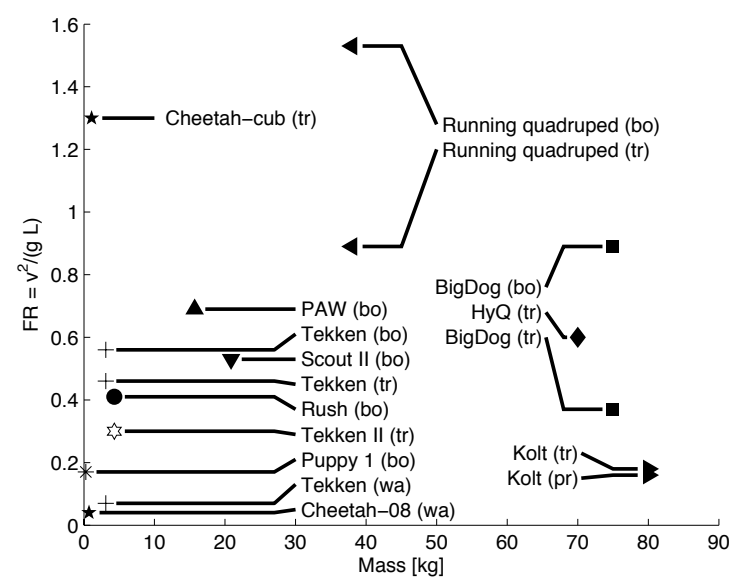

Figure 3: (Literature review) Froude number values of quadruped robots of different mass, all robots were implemented in hardware. wa, bo, tr, pr refer to walk, bound, trot, and pronk, respectively. This is not an exhaustive list of quadruped robots, references and details to the plotted robots are given in Table 1. Froude number is calculated according to (Alexander 1989), equation 14. A recent, however not scientifically published video from the Boston Dynamics' Cheetah reports a bounding or galloping gait speed of $8 \mathrm{~m} / \mathrm{s}$, which would present a Froude number higher than 10-far off this scale.

DOF robots are SCOUT (Buehler et al. 1998), SCOUT II (Battaglia 1999), or the quadruped robot by Iida et al. (2005) with two-segment legs.

Two-segment leg designs of quadruped robots, for example found in Tekken (Fukuoka et al. 2003), KOLT (Estremera et al. 2008) or StarlETH (Hutter et al. 2012), have four possibilities for the orientation of knee and shoulder joints. Indicators for self-stability through leg configuration have been published (Lee et al. 2005). Lee et al. (2005) modeled a trotting quadruped robot with two out of four two-segment leg configurations. Firstly in x-configuration, where the knee joints point inwards, and secondly in o-configuration, where the knee joints point outwards. They report better self-stabilizing pitching behavior for the $\mathrm{x}$-configuration. The directional compliance of the robot leg configuration influenced the orientation of the ground reaction forces (GRF) during stance phase. For the legs in x-configuration, the GRF were rotated closer towards the robot's center of mass (CoM). Fischer et al. (2006, p. 944, Fig. 5) emphasize that by using three segments "multiple limb postures for one leg length" are possible. Yet, only few mammalian-like, quadruped robots have been implemented with three-segment legs (Witte et al. 2001; Iida et al. 2004; Buehler et al. 2005). In mammalian animals, three-segment limb construction is a prominent feature for hind legs, excluding the additional foot segment. The three-segment design is less obvious for mammalian front limbs. Halbertsma (1983); Witte et al. (2002); Fischer et al. (2006) report on the "functional correspondence" of the mammalian scapula (proximal segment of the front leg) to the femur (proximal segment of the hind leg). Fischer et al. (2006) report on scapula step length contribution during walk and trot between $43 \%$ and $73 \%$, for sixteen animals of sizes between short-tailed opossums and elephants. Hence for mammalian quadruped animals, the scapula as the proximal segment of the forelimb "... has 
the major contribution to progression" (Fischer et al. 2006, p. 939). It should be noted that the scapula of a mammal is not fixed by a rotational, skeletal joint, but by a complex of muscles allowing for translations in addition to rotations (Fischer et al. 2006). A robotic hinge joint presents a strong simplification.

Witte et al. (2001) suggested the pantograph leg design, for legged robots, and proposed a pantograph structure which extracts main mammalian leg features, like three-segmentation, segment alignment, and compliant elements. We used these elements as a starting point for the leg design of Cheetah-cub. A compliant pantograph implementation for the front and hindlimb is depicted in Fig. 4c.

Besides the choice for passive, active, or actively supported compliance, the topology and placement of compliant elements is of importance. For mammals, Alexander (1984a) reported elastic energy storage during stance phase. Interstance storage and release mostly appeared in joints distal to the knee, such as hamstring or ankle joints. Gregersen et al. (1998) reported similar results for leg joints of dogs. They found that more than $95 \%$ of the positive work of wrist and ankle extensors, and of the elbow joints could be recoiled through elastic mechanisms during trot gait, but less during gallop. They also discussed that biarticulate leg muscles may be responsible for intra-leg energy transfer.

The spring loaded inverted pendulum (SLIP, Blickhan 1989) was introduced as a compliant extension for dynamic locomotion for the inverted pendulum (IP, Cavagna et al. 1977) model. The SLIP model features a springy, telescopic leg and describes its dynamic, fast running locomotion with flight phases (Blickhan 1989, single leg support) or locomotion during walking (Geyer et al. 2006, short-time double leg support). Like any model, the original SLIP model uses simplifications, such as zero swing leg dynamics, massless legs, an energy conservative model, and a body which is fixed at its hip joint. SLIP predicts self-stabilization through a range of angles of attack (AOA), and system energies. Computed regions of self-stability could predict roughly experimental data from running humans (Seyfarth et al. 2002). SLIP has also been used to predict and explain data from robotic experimentation (Altendorfer et al. 2001). Using SLIP, Poulakakis et al. (2006); Poulakakis et al. (2005) derived a leg angle controller for the Scout robot.

We focus on a single SLIP-like feature, which is the hip joint movement during stance phase, or the corresponding leg length change. Firstly, we can roughly assume that during quadruped trot gait, two legs have a synchronous stance phase. Raibert et al. (1986) combined two synchronously working legs into a single virtual leg, at the center of the robot. For such a virtual leg, the right amount of system energy and angle of attack during stance, SLIP predicts a concave height trajectory of the hip joint. This is opposite to the convex hip movement of a stiff, inverted pendulum-like leg. The leg spring of a SLIP leg in the "original SLIP" fast-running regime will be compressed solely by the robot's momentum. To preserve a SLIP-like hip trajectory during slower robot speeds, we add energy by actively compressing the leg spring at mid-stance. In sum, the dynamic leg compression through body momentum and a leg length actuator can be used to derive an adapted, speed dependent, open loop leg length controller for stance phase contact.

Herr et al. (2000) reported intrinsic pitching stabilization for a modeled horse, in trot gait. The authors identified foot velocity as the major selfstabilizing feed-forward control parameter. The authors also mention the im- 
portance of a passive compliant, neck joint for the modeled horse head in their model.

Daley et al. (2006) designed an experiment with running birds. Guinea fowls stepped at running speed on a paper-covered, hidden pothole, and broke through. Daley et al. (2006) identified that stabilization of the first step into the pothole was led by feed-forward control, and dynamics of the mass-spring system.

Above examples show that dynamic running, and its stability, is not only a product of actuators, skeletal structure, and feedback. Feedback signals and their resulting action responses are restricted in animals through maximum axonal conduction velocity, and conduction delays (More et al. 2010). Nature found solutions for high-speed and high-frequency runs, despite adverse circumstances such as sparse and delayed feedback signals.

Our goal was to find a quadruped robot design and its corresponding open loop controller. We were focusing on a hardware design which required only a minimum of stabilizing control - for trot gait locomotion on flat terrain we ideally would like to run the robot with open loop patterns. We conducted all locomotion experiments with a central pattern generator $(\mathrm{CPG})$ network in open loop mode, i.e. only with inter-oscillator coupling, and no sensory feedback. Oscillator outputs were applied as position signals at the robot's active joints. CPG are capable of more than open loop trajectory generation. Coupled oscillators can take in feedback terms from the environment (Fukuoka et al. 2003; Watanabe et al. 2009). We decided for a CPG, and against a simple sine wave controller. With this choice, we had direct access to additional features such as smooth transient behavior for parameters changes e.g. during start-up of the robot. This avoided jerky robot movements, increased RC servo motor life time, and enabled smoother robot starts. Further, our CPG network required a low number of explicit driving parameters, i.e. only a few parameters had to be optimized. CPG are dynamically self-synchronizing, therefore we were starting experiments with random initial conditions. Finally, at future work we can easily extend our CPG network by adding sensor feedback nodes. Oscillator nodes within a coupled CPG network are coupled to other oscillators by inter-oscillator coupling. Hence there always exists an internal feedback of state information. Here, we refer to the proposed CPG setup as open loop, since no external sensor information is applied.

CPG networks (Taga 1994; Kimura et al. 2007; Ijspeert 2008) have been established for robotic tasks involving rhythmic tasks (Righetti et al. 2009; Narioka et al. 2011) and discrete tasks (Degallier Rochat et al. 2011), with open loop control (Ijspeert et al. 2007) or by using feedback mechanisms (Righetti et al. 2008; Umedachi et al. 2010; Sato et al. 2011). A strong indicator for open loop control in animals was presented in fictive locomotion experiments with isolated lamprey spinal cords (Ijspeert et al. 2007).

\section{Materials and methods}

We describe hardware details of the Cheetah-cub robot (Section 3.1), and explain two tested leg designs. The Webots-Cheetah-cub, the simulated robot, is described in Section 3.2. Hardware and simulated robot are both controlled by the same CPG controller (Section 3.3). We used multiple tools to characterize 
Table 2: Characteristics of the Cheetah-cub robot, in hardware, and simulation. The robot was power-tethered in all experiments.

\begin{tabular}{ll} 
Characteristic & Value \\
\hline $\mathrm{M}_{\text {robot }}$ & $1100 \mathrm{~g}$ \\
$\mathrm{M}_{\text {actuators }}, \mathrm{sum}_{l 3}, \mathrm{M}_{\text {foot }}$ (front) & $590 \mathrm{~g}$ \\
$\mathrm{M}_{l 1}, \mathrm{M}_{l 2}, \mathrm{M}_{l 3}, \mathrm{M}_{\text {foot }}$ (hind) & $11 \mathrm{~g}, 24 \mathrm{~g}, 4 \mathrm{~g}, 6 \mathrm{~g}$ \\
$\mathrm{M}_{l 1}, \mathrm{M}_{l 2}, \mathrm{M}_{l 3}, \mathrm{~g}$ & $12 \mathrm{~g}, 24 \mathrm{~g}, 3 \mathrm{~g}, 6 \mathrm{~g}$ \\
$\mathrm{l}_{\text {hip }}$, standing height & $0.158 \mathrm{~m}$ \\
$\mathrm{~d}_{\text {shoulder-shoulder }}$ & $0.1 \mathrm{~m}$ \\
$\mathrm{~d}_{\text {hip-shoulder }}$ & $0.205 \mathrm{~m}$ \\
RC servo motor & Kondo KRS2350 ICS (8x) \\
Control board & RoBoard RB110 \\
Operation system & Linux Xenomai \\
Communication & Wifi card Via VT6655 \\
Power supply, tethered & $8 \mathrm{~V}$ to $14 \mathrm{~V}$ \\
Length foot $l_{\text {foot }}$ & $14 \mathrm{~mm}$ \\
Stall torque RC servo & $2 \mathrm{Nm} \mathrm{at} 6 \mathrm{~V}$ \\
Speed max RC servo & $0.16 \mathrm{~s} / 60 \mathrm{deg}$ at $6 \mathrm{~V}$ \\
$\mathrm{k}_{\text {diagonal }}$ & $2300 \mathrm{~N} / \mathrm{m}$ \\
$k_{\text {second,front }}$ & $4800 \mathrm{~N} / \mathrm{m}$ \\
$k_{\text {second,hind }}$ & $2330 \mathrm{~N} / \mathrm{m}$ \\
time step SLP, ASLP & $2 \mathrm{~ms}, 0.2 \mathrm{~ms}$ (Webots model) \\
Coulomb friction ground-feed & $1(\mathrm{Webots}$ model) \\
Spring damping & $150 \mathrm{~N} / \mathrm{ms}$ (Webots model) \\
\end{tabular}

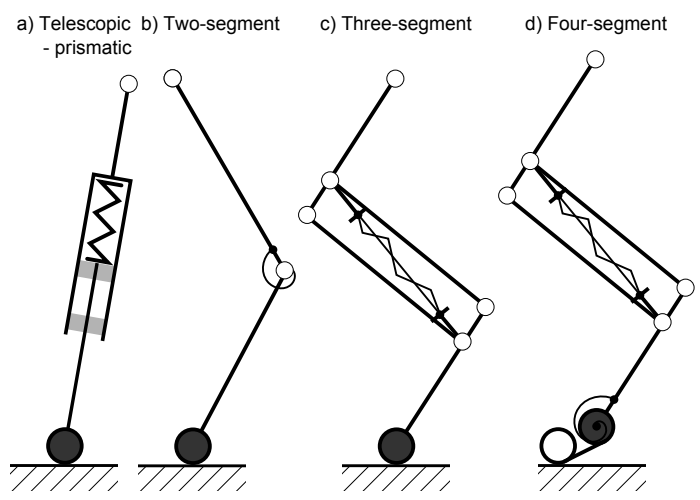

Figure 4: Possible designs for legged robots. a) Telescopic/prismatic leg design. The touch-down point and hip joint are connected with a telescopic mechanism. Here, a passive spring is extending the leg against gravity. Other types of actuation are possible, such as hydraulic or pneumatic cylinders. b) Two-segment leg design, depicted with a torsion spring. c) Three-segment leg design, here shown as a pantograph mechanism, and a gravity-compensating, compression spring mechanism. d) By adding a foot segment to the previous design, a four-segment leg is created. The foot is here coupled with a torsion spring. In the work of this paper, we focus on the three-segment and the four-segment leg design.

and analyze the resulting gaits. The experimental apparatus is described in Section 3.4.

\subsection{Hardware design}

We roughly dimensioned the robot according to a mammalian animal of approximate size and weight of a house cat (Table 3). As suggested by Witte et al. (2003), we based our robot-leg designs on a pantograph mechanism. Here 
Table 3: Segment length ratios per full leg length, for cat-like animals (Felidea) and the Cheetah-cubrobot, based on a three-segment limb. Numbers for Felidea are approximated from (Schmidt et al. 2009, Fig. 4, p. 757 and Fig. 2, p. 752). Hand and foot values are used as given in (Schmidt et al. 2009), values are calculated based on a four-segment limb. The mechanical implementation differs, to avoid front leg/hind leg self collision, and to implement the leg with reasonable effort.

\begin{tabular}{lccc} 
& Felidea & Cheetah-cubrobot & \\
\hline scapula & 0.28 & 0.26 & $l_{1}^{\mathrm{f}}$ \\
humerus & 0.38 & 0.42 & $l_{2}^{\mathrm{f}}$ \\
radius & 0.35 & 0.32 & $l_{3}^{\mathrm{f}}$ \\
femur & 0.38 & 0.41 & $l_{1}^{\mathrm{h}}$ \\
tibia & 0.37 & 0.42 & $l_{2}^{\mathrm{h}}$ \\
tarsometatarsus & 0.245 & 0.17 & $l_{3}^{\mathrm{h}}$ \\
hand/foot & 0.12 & 0.08 & $l_{\text {foot }}$
\end{tabular}

a) SLP leg

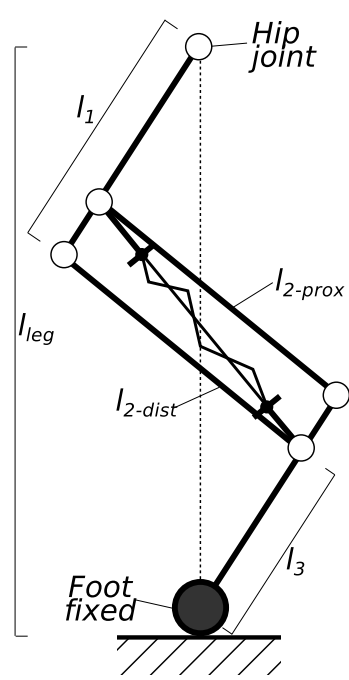

b) ASLP leg

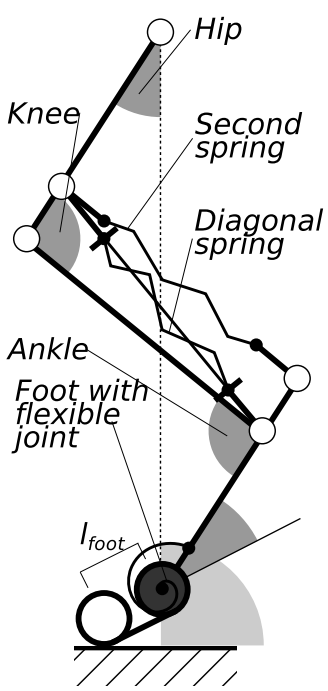

Figure 5: Schematic presentation of the two proposed leg designs: spring loaded pantograph (SLP, left) and advanced SLP (ASLP, right). Both are used in the following for quadruped locomotion experiments in simulation and hardware. Detailed segment lengths of the hind and front leg are provided in Table 3.

we propose two leg designs: a first design with a three-segment, spring loaded pantograph leg (SLP-leg). And a second, four-segment advanced spring loaded pantograph leg design (ASLP-leg), with in-series elastic elements.

Spring loaded pantograph leg (SLP leg) The proximal $\left(l_{1}\right)$ and distal $\left(l_{3}\right)$ limb segments of the spring loaded pantograph leg (SLP, Fig. 5) are connected with a parallel mechanism. This keeps the $l_{1}$ and $l_{3}$ segments parallel (Fig. 5). The pantograph design resembles mammalian animals' leg segment behavior for most part of a stride cycle: swing phase, heel down and mid-stance (Fischer et al. 2006). A cylinder shaped foot element, covered with duct tape, is mounted. 


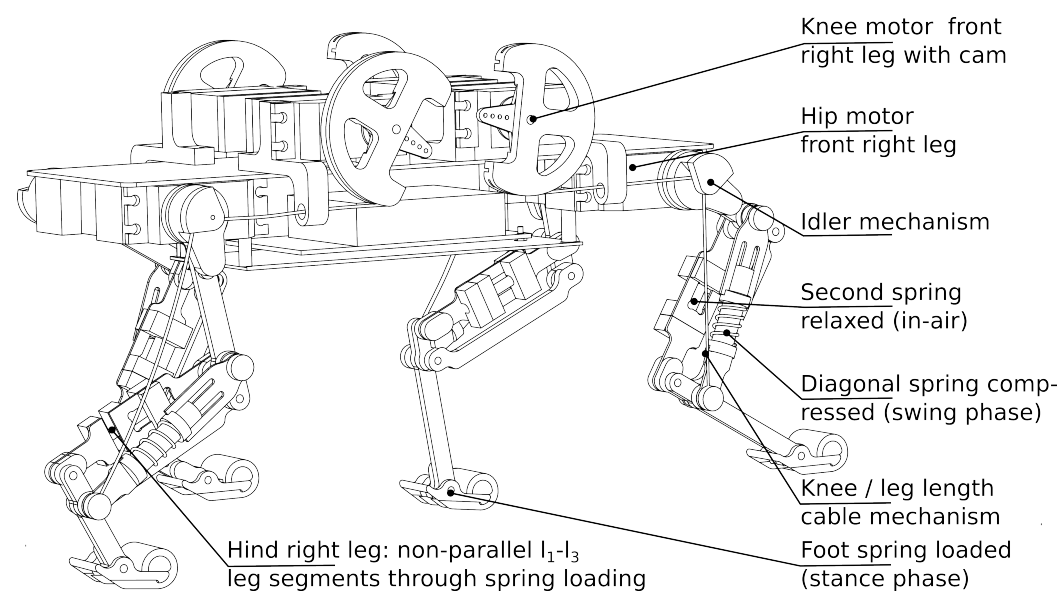

Figure 6: Cheetah-cub robot, view from hind right, the robot's front is to the right. Front right and hind left leg are in swing phase, front left and hind right are in stance phase. The leg's cable mechanism goes from the knee motor cam, via the idler pulley at the hip joint, and is attached at the lower end of the diagonal spring. The low radius idler pulley (diameter $1 \mathrm{~mm}$ ) decoupled almost fully knee and hip motor movements. This allowed the proximal mounting of both actuators.

Robot legs are equipped with a diagonal spring mechanism (Fig. 5). The diagonal spring spans over the $l_{2}$ segment (Fig. 5,6 ), and exerts a leg-extension force at all times. In sum, the SLP leg design classifies as a passively extending, gravity loaded, compliant leg.

Advanced spring loaded pantograph leg (ASLP leg) Pantograph behavior reflects the $l_{1}-l_{3}$-angular relationship in animals well, but not between mid-stance and toe off. During this time, the distal $\left(l_{3}\right)$ segment deflects further, due to high external load acting on its elastic elements (Fig. 6). For the ASLP leg, a biarticulate spring element ("second spring", Fig. 5) was implemented as a replacement of the rigid pantograph $l_{2-\text { prox }}$ segment (Fig. 5 , hind right leg Fig. 6). An in-series spring-loaded foot element replaced the cylinder shaped foot design of the SLP leg.

Leg actuation and robot body The robot's legs are each actuated by two RC servo motors (Table 2), both actuators are mounted proximally. The knee/elbow actuator is attached to the body's center. It actively flexes the leg via a cable mechanism, as an antagonist to the diagonal spring. This cable mechanism acts as an automatic decoupling mechanism, it goes slack if external forces compress the leg. The general leg length actuation and decoupling by a cable mechanism was implemented earlier, for example with the Bow leg (Zeglin 1999), however with a different leg design, and cable actuation. Cheetah-cub robot's hip/shoulder actuator is directly mounted between body and leg. It protracts (swings forward) and retracts (swings backward) the front or hind leg.

The robot's body is stiff, and made from thin carbon fiber sheets. For all experiments, the robot was tethered for power supply. CPG computation, RC 

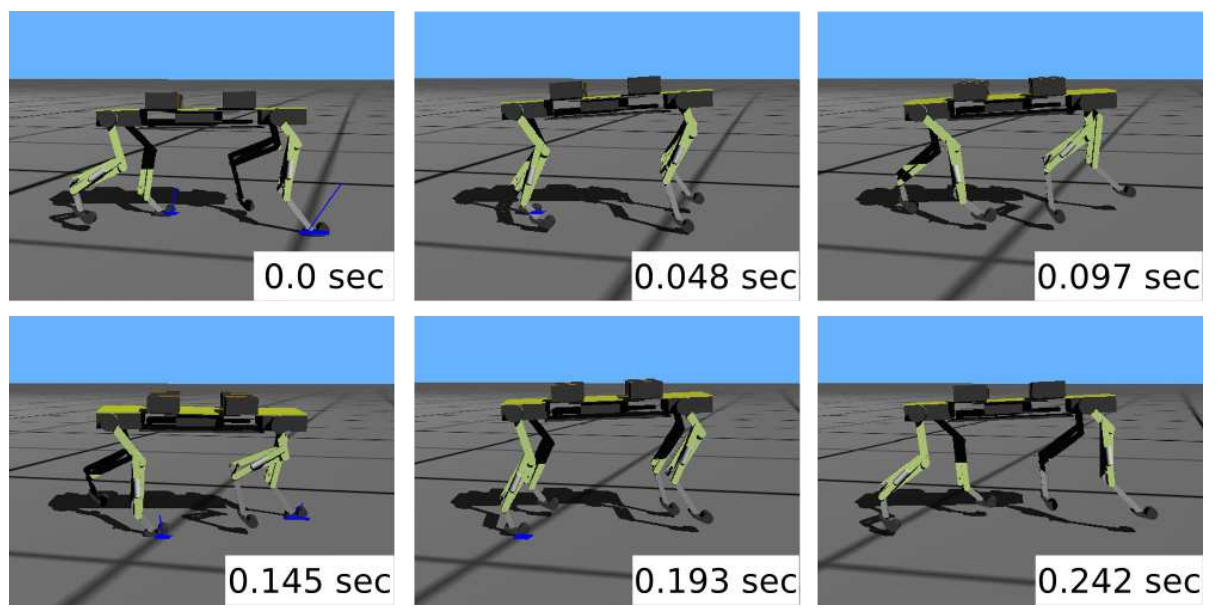

Figure 7: (Webots simulation) Snapshots of one gait cycle of the Cheetahcub robot in trot gait. Simulation environment was Webots. The robot was trotting freely with an open loop control at a frequency of $3.5 \mathrm{~Hz}$. The mean horizontal locomotion speed was $1.14 \mathrm{~m} / \mathrm{s}$. Each speed measurement run lasted $15 \mathrm{~s}$.

servo motor signal generation, and wireless communication are controlled from a RB110 single board computer, mounted on the robot's body.

\subsection{Webots model description}

A simulated model of the Cheetah-cub robot was created in Webots (2009), for both leg configurations, before testing the hardware platform. This allowed us to use extensive optimization runs with different trot-gait parameters, and without hardware wear-down. By applying stochastic optimization for parameter tuning, we expected to get insights about the required robot control parameters.

Simulation results were collected by running a Webots model of the Cheetahcub robot (Fig. 7). Control parameters were optimized with particle swarm optimization (PSO, Eberhart et al. 1995). 60 particles were used for both leg configurations, for 400 iterations, and 10 frequencies from $1.25 \mathrm{~Hz}$ to $3.5 \mathrm{~Hz}$ in steps of $0.25 \mathrm{~Hz}$ (Fig. 9). The simulated robot model was implemented according to the hardware robot (Table 2). A video of the robot modeled and running in Webots can be found in Extension 2 (third part). Calculation time depended on the complexity of the simulation model. A single simulation run of a SLP-leg took an average of $t=0.5 \mathrm{~min}$, and a simulation run with an ASLP leg required $t=2.5 \mathrm{~min}$. The simulated run time was $20 \mathrm{sec}$. We used a 40 core computer cluster (Intel Xeon CPU, model E5504 at $2 \mathrm{GHz}, 1$ Gbyte memory per core) for parallel computation. Simulation of both robot configurations took 12.5 days, in total. The fitness function $f(x)$ was chosen to optimize the average forward speed of the robot over a fixed time window. Vector $x$ contained CPG control parameters, similar to Spröwitz et al. (2008).

All simulation runs were started with the robot standing on the ground, with roll-, pitch-, and yaw-angle, and the body's sideways translation DOF fixed. After the robot accelerated for $3 \mathrm{sec}$, the restriction was released, and the 
robot system ran freely for another $2 \mathrm{sec}$ of entrainment time. The remaining $15 \mathrm{sec}$ of free running were used for speed recording. If the robot fell, a zero speed value was returned to the fitness function. Standard Webots friction and damping values were applied (Table 2).

\subsection{Control}

Central pattern generators (CPG) were successfully implemented to generate locomotion patterns for legged and other robots (Fukuoka et al. 2003; Ijspeert 2008; Spröwitz et al. 2008). Here, we apply CPG to easily parameterize and synchronize a set of open loop trajectories. In the future, we will also use CPG to close the sensor/actuator loop.

The hip joint driving CPG consists of a fully connected network of four phase-coupled oscillators, each oscillator drives one hip joint. A glossary of the applied CPG parameters is available in Table 4.

Table 4: Glossary of applied CPG parameters, and hardware abbreviations.

$$
\begin{aligned}
& \begin{array}{llll}
\text { Abbrv. } & & \text { Abbrv. } & \\
\hline D & \text { duty factor, measured } & \text { CPG } & \text { central pattern generator } \\
D_{\text {vir }} & \text { duty factor, virtual } & \phi_{i} & \text { oscillator linear phase, [rad] } \\
\text { M } & \text { body mass } & \Theta_{i}^{h} & \text { phase of hip joint, [rad] } \\
F g & \text { ground reaction force } & \Theta_{i}^{k} & \text { phase of knee joint, [rad] } \\
\text { MS } & \text { mid stance } & \varphi_{i, j} & \text { desired phase lag between osc. } i \text { and } j,[\mathrm{rad}] \\
\text { HD } & \text { heel down } & O_{i}^{[h, k]} & \text { desired offset for hip or knee i } \\
\text { TO } & \text { toe off } & o_{i}^{[h, k]} & \text { instantaneous offset } \\
\text { SLP leg } & \text { spring loaded pantograph leg } & A_{i}^{h} & \text { desired hip amplitude [rad] } \\
\text { ASLP leg } & \text { advanced SLP leg } & a_{i}^{[h, k]} & \text { instantaneous amplitude for hip or knee i } \\
\text { CoT } & \text { cost of transport [J/(Nm)] } & \mathrm{h}, \mathrm{k} & \text { hip, knee } \\
\text { RC servo } & \text { radio controlled servo motor } & \mathrm{hk}, \mathrm{hh}, \mathrm{fk}, \text { fh } & \text { hind knee, hind hip, front knee, front hip } \\
\mathrm{f} & \text { stride frequency [Hz] } & \Gamma_{i}^{[h, k]} & \text { motor position/ joint angle } \\
\text { O } & \text { offset } & A_{s t}^{k} & \text { desired stance phase amplitude, [ratio] } \\
\text { PSO } & \text { particle swarm optimization } & A_{s w}^{k} & \text { desired swing phase amplitude, [ratio] }
\end{array} \\
& \dot{\phi}_{i}=2 \pi f+\sum_{j \neq i} k_{i, j} \sin \left(\phi_{j}-\phi_{i}-\varphi_{i, j}\right) \\
& \dot{a}_{i}^{h}=\alpha\left(A_{i}^{h}-a_{i}^{h}\right) \\
& \dot{o}_{i}^{h}=\alpha\left(O_{i}^{h}-o_{i}^{h}\right) \\
& \Theta_{i}^{h}= \begin{cases}\frac{\phi_{i}}{2 D_{\text {vir }}} & 0 \leq \phi_{i} \leq 2 \pi D_{\text {vir }} \\
\frac{\phi_{i}+2 \pi\left(1-2 D_{\text {vir }}\right)}{2\left(1-D_{\text {vir }}\right)} & \end{cases}
\end{aligned}
$$

The virtual duty factor $D_{\text {vir }}$ is the fraction of time where the leg moves rearwards, of the full cycle duration. Virtual duty factor is a control parameter that should be distinguished from the actual duty factor $D$. The latter describes in animal gaits the externally measured "... fraction of stride time $(T)$ that a limb is in contact with the ground" (Biewener 1983). $D_{\text {vir }}$ differs from $D$ in the case of body pitching movements.

Hip joint motor command $\Gamma_{h}$ is derived as:

$$
\Gamma_{i}=a_{i}^{h} \cos \left(\Theta_{i}^{h}\right)+o_{i}^{h}
$$

During swing phase the controller actively reduces leg length to avoid collision between the swinging foot and the ground. During mid stance phase, knee 
stance phase deflection $A_{\mathrm{h}, \mathrm{st}}$ can shorten leg length. This parameter aims to decrease vertical hip oscillations of the stance leg to reduce the robot's roll- and pitch-movements. Knee deflections (swing phase: $A_{\mathrm{sw}}^{\mathrm{k}}$, stance phase: $A_{\mathrm{st}}^{\mathrm{k}}$ ) are defined dimensionless, as a fraction of leg length. We set the knee phase to:

$$
\Theta_{i}^{k}=\Theta_{i}^{h}+\varphi_{h, k}
$$

The knee joint motor receives $\Gamma_{k}$ as follows, the knee joint profile adjustment is made using a piecewise cubic profile:

$$
\begin{aligned}
a_{i}^{k} & =\left\{\begin{array}{c}
A_{s t}^{k}, \text { if } \Theta_{i}^{k}<\pi \\
A_{s w}^{k}, \text { if } \Theta_{i}^{k} \geq \pi
\end{array}\right. \\
\dot{o}_{i}^{k} & =\alpha\left(O_{i}^{k}-o_{i}^{k}\right) \\
\theta_{i}^{\prime} & \equiv \frac{\Theta_{i}^{k}}{2 \pi}(\bmod 0.5) \\
\theta_{i} & =2 \cdot \theta_{i}^{\prime} \\
\gamma_{i} & =\left\{\begin{array}{l}
-16 \theta_{i}^{3}+12 \theta_{i}^{2}, \text { if } \theta_{i}<0.5 \\
12\left(\theta_{i}-0.5\right)^{3}-12\left(\theta_{i}-0.5\right)^{2}+1
\end{array}\right. \\
\Gamma_{i}^{k} & =a_{k}^{i} \gamma_{i}+o_{i}^{k}
\end{aligned}
$$

The resulting open loop trajectories are plotted for one stride cycle in Fig. 8.

\subsection{Data Collection}

This section describes the hardware setup and software tools used to capture gait characteristics of the hardware robot. We recorded in hardware 6 DOF (fully defined rigid body in space) motion capture data (MOCAP), ground reaction forces (GRF), high-speed video footage, and power consumption. Kinematic data of the robot was recorded by a MOCAP system based on infrared reflective markers (11 mm diameter). Twelve MOCAP cameras (Optitrack s250e, Naturalpoint, Inc. 2011) were mounted at $1.50 \mathrm{~m}$ and $2.50 \mathrm{~m}$ height, positioned in a large rectangle around the catwalk (Fig. 2). Cameras observed a volume of $1 \mathrm{~m}$ width, $4 \mathrm{~m}$ length, and $0.5 \mathrm{~m}$ height. Data was captured at $f=240 \mathrm{fps}$, with a precision of about $1 \mathrm{~mm}$. A ground plane calibration provided an absolute coordinate base frame. Collected marker trajectories were processed and cleaned by using Arena (Naturalpoint, Inc. 2011) software, and exported in c3d-format. Markers were labeled and ordered in Mokka (Barre et al. 2011), data was loaded with Matlab (MATLAB 2009, v. 7.9) with the help of $b$-tk plug-in (Barre et al. 2011). MOCAP data was low-pass filtered with a cut-off frequency of $18 \mathrm{~Hz}$.

Ground reaction forces (GRF) were measured by two side-by-side mounted force plates (type 9260AA3, Kistler 2011). Force plate (FP) signals were sampled by an A/D converter (Kistler Bioware 64ch DAQ-System, type 5695A1) at $f=1000 \mathrm{~Hz}$. Force plate surfaces were coated with paper tape, to avoid reflections of the infrared LEDs from the tracking system. The catwalk was assembled from wooden boards, the force plates were flush mounted with the boards. The friction coefficient of the wooden board and the paper-taped force plates was about equal.

A current probe clamped around the power cable measured the robot's power consumption. A digital oscilloscope (LeCroy 6100) sampled the amplifier output 


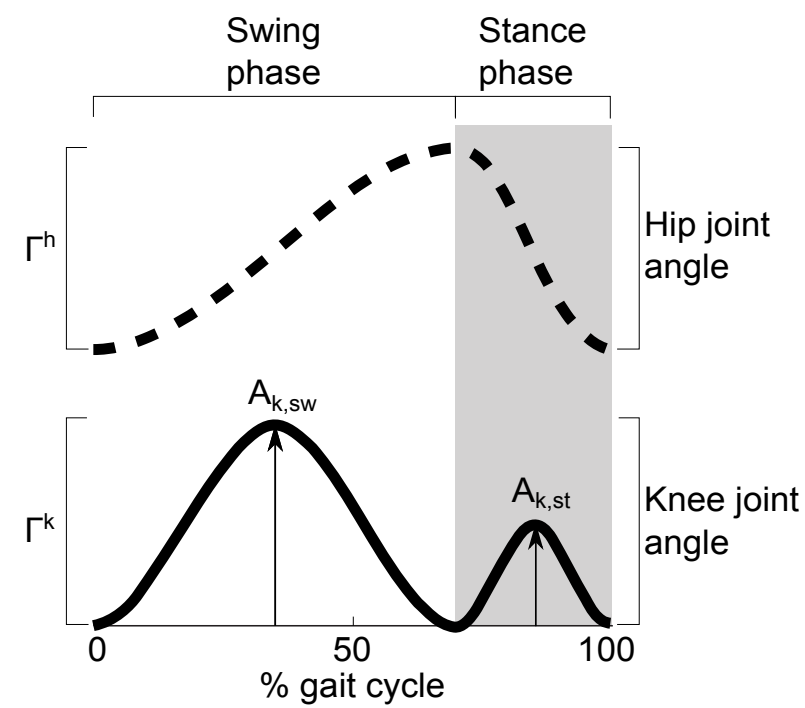

Figure 8: Control signals for servo motor position control: hip angle (top, dashed line) and knee angle (bottom, solid line) for one stride cycle. Hip signal (Eqn. 4) enables direct duty factor setting, hence the hip signal is a skewed sine wave. Swing phase is plotted on the left side, stance phase on the right side. The knee signal is two peaked, the larger peak refers to swing leg deflection $A_{\mathrm{sw}}$. This shortens robot legs during (forward) swing phase, to avoid ground collision. The second, smaller knee signal peak $\left(A_{\mathrm{st}}\right)$ is the knee motor activation during stance phase. Active leg shortening overlaps with the passive leg shortening from forces along the leg axis in stance phase, it was implemented to decrease vertical hip movement.

(Tektronix TM502A, AM 5033) at $f=50 \mathrm{kHz}$, data was stored digitally, filtered in Matlab, and low-pass filtered at $18 \mathrm{~Hz}$. The voltage of the power supply was directly read with a voltmeter. Standby power consumption of the RB110 control board and the RC servo motors $(4 \mathrm{~W})$ was subtracted from all measurements.

Initial recording trials of infrared markers mounted on leg joints were dismissed. The markers were too close to each other and produced faulty data in the relatively large capture volume. High-speed video footage was recorded instead, at $f=240 \mathrm{~Hz}$. The camera (Casio camera, model EX-ZR100) was mounted perpendicularly to the catwalk, either fixed, or on a rail manually following the robot. Joints were tracked from video footage using Tracker software (Brown 2012), the data was processed in Matlab.

MOCAP, GRF, and video footage data were synchronized by tapping a wooden hammer on the GRF plates, while recording it with a high-speed camera. The hammer had a single MOCAP marker mounted, that the MOCAP system recorded simultaneously. Data was synchronized manually in Matlab with an accuracy of $t \approx 20 \mathrm{~ms}$, after sampling it at $f=1000 \mathrm{~Hz}$.

In Section 4.2, data from 115 experimental runs is presented. Gaits were marked as successful when the robot was able to cross the catwalk ( $4 \mathrm{~m}$ length, Fig. 2a), during trotting with no restraints, with a power cable attached and 


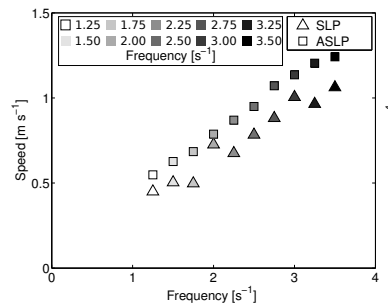

(a)

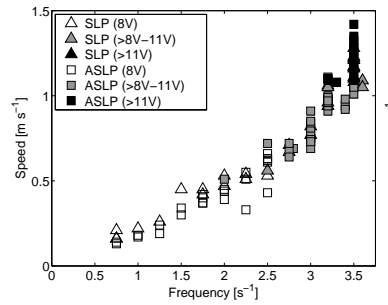

(d)

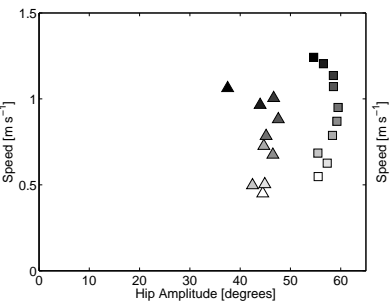

(b)

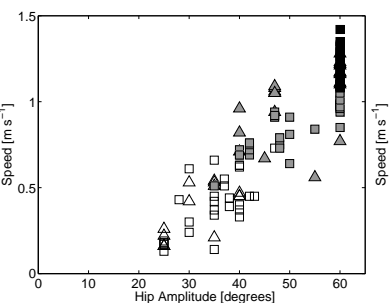

(e)

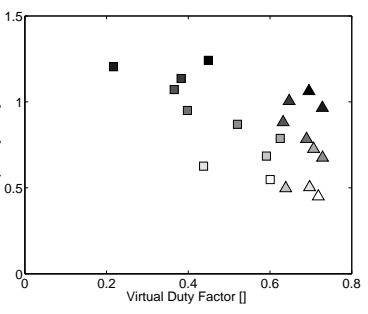

(c)

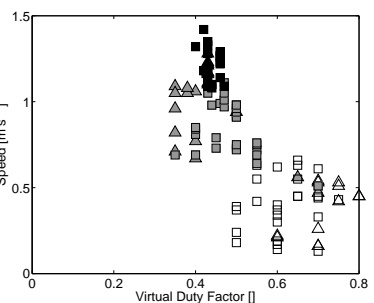

(f)

Figure 9: Top row: (Webots simulation). Twelve frequencies between $f=$ $1.5 \mathrm{~Hz}$ and $f=3.5 \mathrm{~Hz}$ are shown, with increasing grey-color as in Fig. 9a. Symbols as in Fig. 9a. Bottom row: (Hardware experiment). Symbols of the hardware column are as in Fig. 9d, color-coding is according to the chosen motor voltage.

kept loose carefully. Gaits reached step lengths of up to $l=0.28 \mathrm{~m}$. Therefore, a typical trot gait cycle lead up to 25 steps per experimental run. For experiments, the robot was started while in the air, and set down manually onto the catwalk. It was intercepted manually before hitting the end of the catwalk. Robot speed was measured covering a locomotion distance between $1.2 \mathrm{~m}$ to $3 \mathrm{~m}$, depending on the robot speed. For speed recording, we waited for the robot to reach steady state locomotion $(0.5 \mathrm{~s}$ to $1 \mathrm{~s})$.

Cost of transport (Gabrielli et al. 1950; Tucker 1970; Kuo 2007, CoT) is calculated as:

$$
C o T=P_{\mathrm{el}} /(M \cdot g \cdot \bar{v})
$$

with $P_{\mathrm{el}}$ being the electric power used for actuation, $M$ the robot's mass, and $\bar{v}$ the mean forward robot speed. CoT is dimensionless, or here for clarity in $\left[\mathrm{J} \mathrm{N}^{-1} \mathrm{~m}^{-1}\right]$. Froude number (Alexander 1989; Alexander 1996) is calculated using acceleration of Earth gravity $(g)$, mean forward velocity $(\bar{v})$, and the hip joint height of the standing robot $(h)$.

$$
\mathrm{FR}=\bar{v}^{2} /(g \cdot h)
$$

Froude number is a dimensionless number, it enables size-independent comparison of animals and robots in terms of speed. 


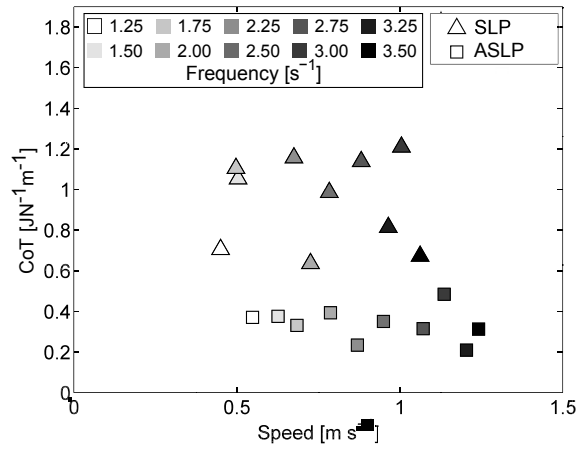

(a) Webots simulation.

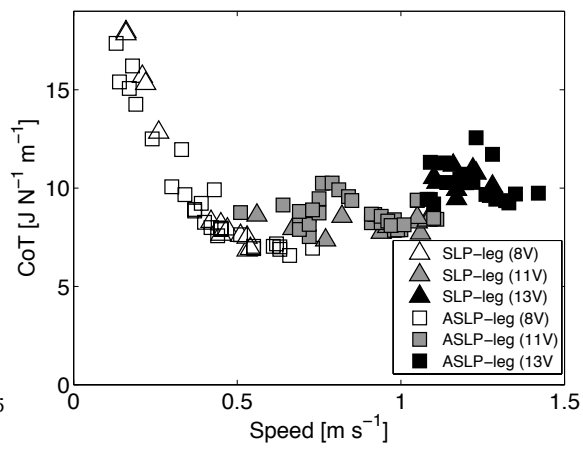

(b) Hardware experiment.

Figure 10: Cost of transport versus robot speed. Simulation-CoT is more than 15 times less than in hardware. This is likely due to gearbox losses of the high-geared RC servo motors (ratio $300: 1$ ).

\section{Results}

We conducted quadruped locomotion experiments in simulation (Section 4.1) and with the Cheetah-cub hardware platform (Section 4.2). In both environments, two leg configurations (SLP and ASLP) were tested. All results reported are based on CPG open loop control. During hardware experiments, we recorded MOCAP data (CoM speed and angles), ground reaction forces plates (GRF), high-speed video footage (joint angles, ground contacts), and electric power to analyze the trot gait runs. Equivalent sensor values were extracted from the Webots simulation. Results of 120 step-down experiments, with 20 runs per configuration, and three step-down heights are presented in Section 4.2.2, where we recorded speed, counted successful runs, and recorded video footage.

\subsection{Webots Simulation Results}

Figures 9a-9c show results from Webots simulation for both configurations SLP and ASLP. Stride cycle frequencies were fixed between $f=1.25 \mathrm{~Hz}$ and $f=3.5 \mathrm{~Hz}$, in steps of $0.25 \mathrm{~Hz}$. The particle swarm optimization found many valid robot gaits, for both robot leg configurations, and over a large range of input parameters and speeds. Here, every sample point represents average values of the best (fastest, producing a stable gait) 100 particles per frequency.

Speed Fig. 9a shows a roughly linear frequency/speed relationship, for both robot leg configurations. SLP robot gaits ranged between $v=0.45 \mathrm{~m} / \mathrm{s}$ and $v=1.06 \mathrm{~m} / \mathrm{s}$, ASLP gaits from $v=0.55 \mathrm{~m} / \mathrm{s}$ to $v=1.22 \mathrm{~m} / \mathrm{s}$. Hence the ASLP configuration showed a $16 \%$ higher top speed. Higher ASLP speed can be explained firstly by a larger leg length at toe-off. Further, the additional $l_{2}$ springs and foot-springs can store energy during stance phase, which is released at toe-off.

Cost of transport (CoT) Fig. 10a shows the CoT behavior plotted against speed, for both simulated robot configurations SLP and ASLP. All positive and 


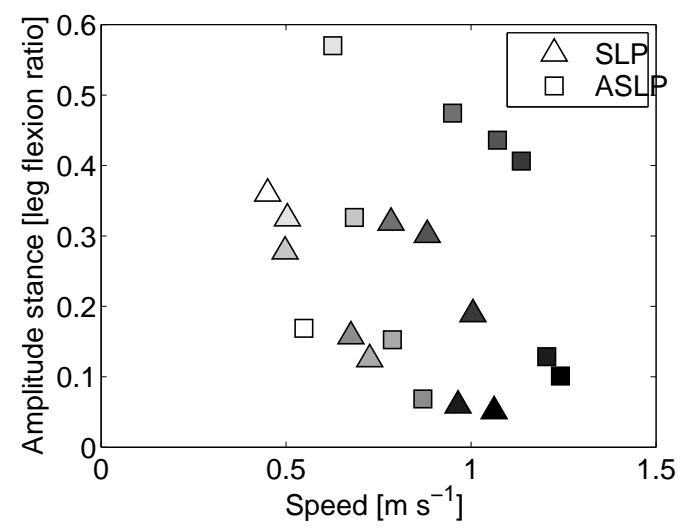

Figure 11: Plot of active stance phase deflection $\left(A_{\mathrm{st}}^{\mathrm{k}}\right)$ versus robot speed, for the simulated robot (SLP and ASLP configuration) optimized with PSO. All successful gaits, found by optimization, applied active knee stance deflection larger than zero. Color coding as in Fig. 9a.

negative forces and torque values were integrated over the running distance, to calculate energy consumption. Energy consumption was normalized into CoT according to equation 13 . The CoT of the SLP configuration was in average $\operatorname{CoT} \approx 1 \mathrm{~J} / \mathrm{N} / \mathrm{m}$, ranging from $0.7 \mathrm{~J} / \mathrm{N} / \mathrm{m}$ up to $1.25 \mathrm{~J} / \mathrm{N} / \mathrm{m}$. CoT of the ASLP configuration decreased slightly with increasing robot speed. It was in average $0.4 \mathrm{~J} / \mathrm{N} / \mathrm{m}$, ranging from $0.25 \mathrm{~J} / \mathrm{N} / \mathrm{m}$ up to $0.54 \mathrm{~J} / \mathrm{N} / \mathrm{m}$. Therefore, ASLP with its additional compliant leg segment and its compliant foot joint was more than double as CoT-efficient as the SLP configuration.

Hip amplitudes Fig. 9b plots the hip amplitudes found by PSO. A clustering of hip amplitudes for both leg configurations is prominent, independent from the stride frequency. SLP hip amplitudes ranged between $38^{\circ}-48^{\circ}$. Lowest ASLP hip amplitudes were found at $55^{\circ}$ at $1.24 \mathrm{~m} / \mathrm{s}$, highest ASLP hip amplitude was $59^{\circ}(v=0.95 \mathrm{~m} / \mathrm{s})$. Therefore, ASLP hip amplitudes were found in a narrow window of less than $4^{\circ}$, around $55^{\circ}$. In sum, PSO found stable gaits with amplitudes $\approx 10^{\circ}$ higher than SLP amplitudes. Roughly $30 \%$ higher hip amplitudes at equal stride frequencies can explain higher speed values of the ASLP robot.

Virtual duty factor Fig. 9c shows plots of robot speed versus virtual duty factor. The virtual duty factor for the robot was set according to equation 4 . SLP gaits had a virtual duty factor in a narrow range, from 0.6 to 0.73 , and barely ever showed flight phases with all feet off the ground (data not shown). ASLP configuration of the simulated robot showed good capability to increase speed by decreasing $D_{\text {vir }}$. High speed values of configuration ASLP were found for virtual duty factors around 0.4 and included short flight phases.

Stance phase leg actuation In Fig. 11, values of $A_{\mathrm{st}}^{\mathrm{k}}$ are plotted. $A_{\mathrm{st}}^{\mathrm{k}}$ indicates the amplitude of active leg shortening through the knee motor during stance phase. SLP robot gaits with a lower speed $(v=0.45 \mathrm{~m} / \mathrm{s})$ used a rel- 

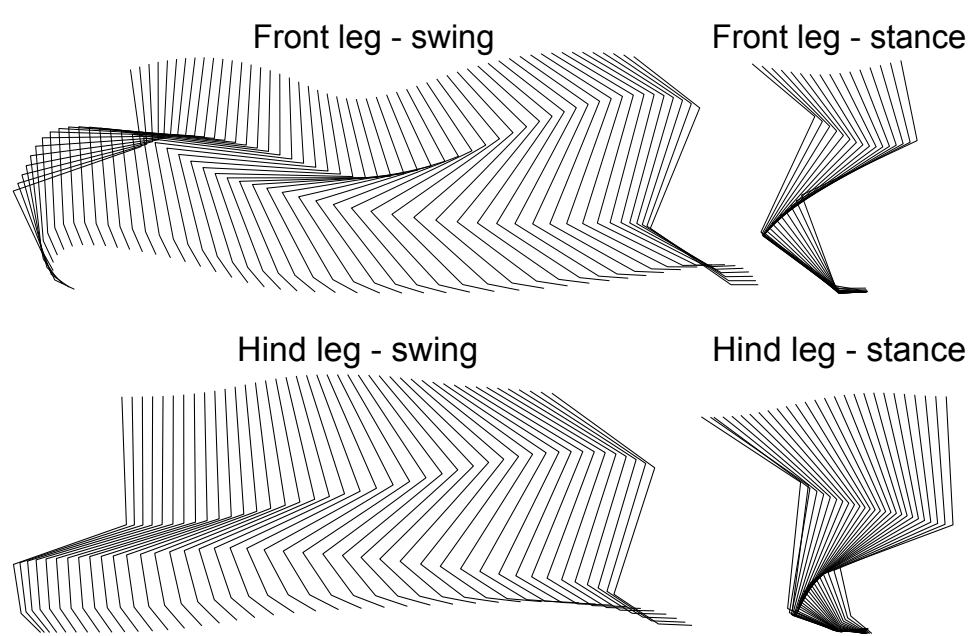

Figure 12: (Hardware experiment) Stick figure sequence of right forelimb and hindlimb, robot in ASLP-configuration. About $t=4.2 \mathrm{msec}$ are between consecutive lines. The speed of the robot is $v=1.24 \mathrm{~m} / \mathrm{s}$, the locomotion cycle frequency is $f=3.5 \mathrm{~s}^{-1}$. The front limb figure shows a SLIP behavior at the shoulder joint with a minimum during mid-stance, but no clear leg flexion profile is visible for the hindlimb. Swing leg behavior of the forelimb shows leg retraction after toe-off, and slight leg retraction before touch-down; features also documented for quadruped mammals (Alexander 1984b).

Table 5: Open parameters for hardware experiments and Webots simulation. From simulation optimization we derived that frequency $f$, and hip amplitude $A^{\mathrm{h}}$ increased linearly with speed. Decreasing duty factor $D$ also increased speed. The remaining parameters were manually, iteratively adjusted to gain stable gaits in hardware. Knee deflection values are expressed as leg deflection ratios, i.e. a value of zero means a fully extended leg and a value of 1 a fully flexed leg.

\begin{tabular}{rllll} 
& Parameters & Abb. & Range & Unit \\
\hline 1 & Frequency & $f$ & $1.25-3.5$ & {$\left[\mathrm{~s}^{-1}\right]$} \\
2 & Duty factor & $D_{\mathrm{vir}}$ & $0.95-0.05$ & \\
3 & Amplitude hip & $A^{\mathrm{h}}$ & $0-60$ & $\left.{ }^{\circ}\right]$ \\
4 & Offset knee & $O^{\mathrm{k}}$ & $0-0.25$ & {$[\mathrm{rad}]$} \\
5 & Offset hip & $O^{\mathrm{h}}$ & $-20-20$ & $\left.{ }^{\circ}\right]$ \\
6 & Defl. stance knee & $A_{\mathrm{st}}^{\mathrm{k}}$ & $0-0.8$ & \\
11 & Defl. swing knee & $A_{\mathrm{sw}}^{\mathrm{k}}$ & $0-1.0$ &
\end{tabular}

atively high stance phase knee deflection of $A_{\mathrm{st}}^{\mathrm{k}}=0.36$. At higher robot speed $(v=1.06 \mathrm{~m} / \mathrm{s}), A_{\mathrm{st}}^{\mathrm{k}}$ was reduced to 0.05 . Maximum $A_{\mathrm{st}}^{\mathrm{k}}$ for the ASLP configuration was $0.57(v=0.6 \mathrm{~m} / \mathrm{s})$. For high-speed ASLP gaits, $A_{\mathrm{st}}^{\mathrm{k}}$-values reduced equally strong as SLP gaits $\left(v=1.24 \mathrm{~m} / \mathrm{s}\right.$ at $\left.A_{\mathrm{st}}^{\mathrm{k}}=0.11\right)$. In summary, for both leg configurations, $A_{\mathrm{st}}^{\mathrm{k}}$ decreased with increasing speed.

\subsection{Hardware Experiments Results}

Simulated gaits could not directly be transferred to hardware, or at least not with the same resulting robot speed. This was likely due to differences in ground 

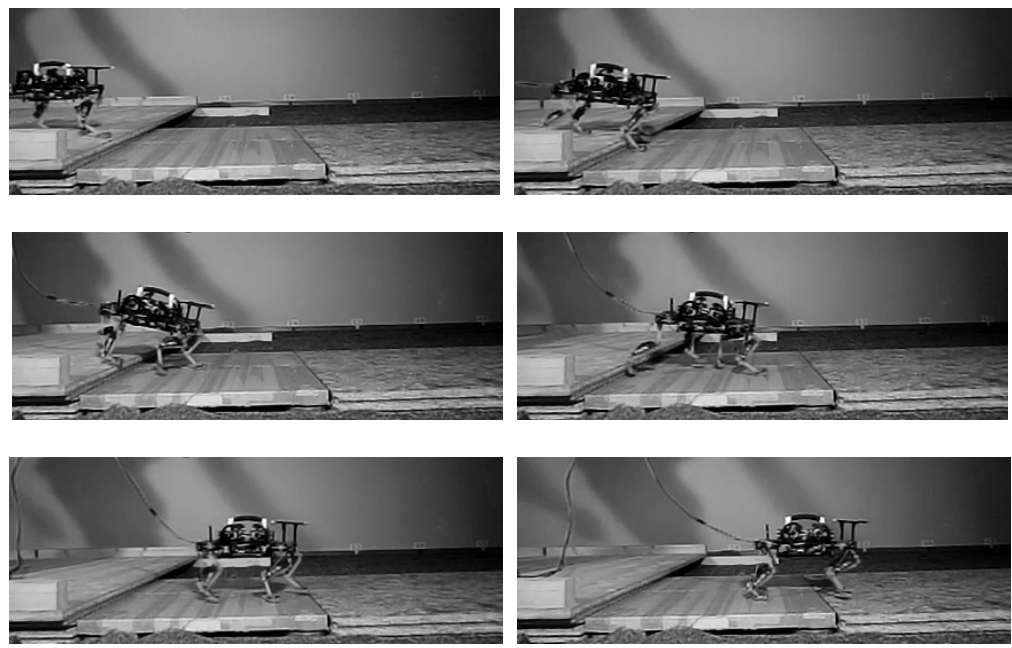

Figure 13: (Hardware experiment) Snapshot series of the robot (ASLP) successfully traversing a step-down perturbation of $32 \mathrm{~mm}$, or $20 \%$ of its standing hip height / leg length. Time difference between pictures is $t=0.105 / \mathrm{s}$. Locomotion direction is from left to right, mean forward robot speed was $\bar{v}_{\text {hor }}=1.1 \mathrm{~m} / \mathrm{s}$. The robot dissipated the additional energy from the vertical drop by small successive vertical body oscillations in the next three to four cycles after touchdown. The corresponding video is shown in Extension 3 (first part).

friction values, damping effects in joints and spring mechanisms, and effects of reflected motor inertia due to high gear ratios. For the simulated quadruped, standard values for ground-friction and damping were applied. Further RC servo motor-speed and motor-torque were transferred, but no gearbox details (inertia, friction losses) were implemented. Instead, we followed an empirical approach of testing key parameter behavior on the hardware robot, which we had identified earlier in simulation (Section 4.1). We expected a linear speed response to increased hip amplitude and locomotion frequency, and a negative-slope linear relation for increasing duty factor values. Remaining parameters (Table 5) were selected and set manually, to stabilize the robot. The starting point for hardware experiments was taken from one stable, fast gait from simulation. This transfer instantly lead to a stable hardware trot gait $(v=0.35 \mathrm{~m} / \mathrm{s})$. For this first hardware parameter characterization, 115 experiments were recorded with successful trot gaits (Fig. 9). Experimentally found CPG parameters (Fig. 9d9f) were mapped into drive functions. The best data fit which also minimized the Euclidean deviation of each CPG parameter set between two similar desired speed values was used to define this set of drive functions (Table 6). We then conducted two further types of experiments. For a single gait of $\bar{v} \approx 1.15 \mathrm{~m} / \mathrm{s}$ we tested both hardware configurations (SLP and ASLP) for their robustness rejecting perturbations for a step-down obstacle. 20 runs per leg configuration and per step-down height were recorded. Finally, simulation results suggested that a linear decrease of $A_{\mathrm{st}}^{\mathrm{k}}$ was best to run the robot with a stable trot gait, from low to high-speed (second hypothesis). We implemented four different $A_{\mathrm{st}}^{\mathrm{k}}$ drive functions (decreasing, constant, increasing, and zero $A_{\mathrm{st}}^{\mathrm{k}}$ ), and characterized them by speed, roll-angles, and pitch-angles over a frequency range 

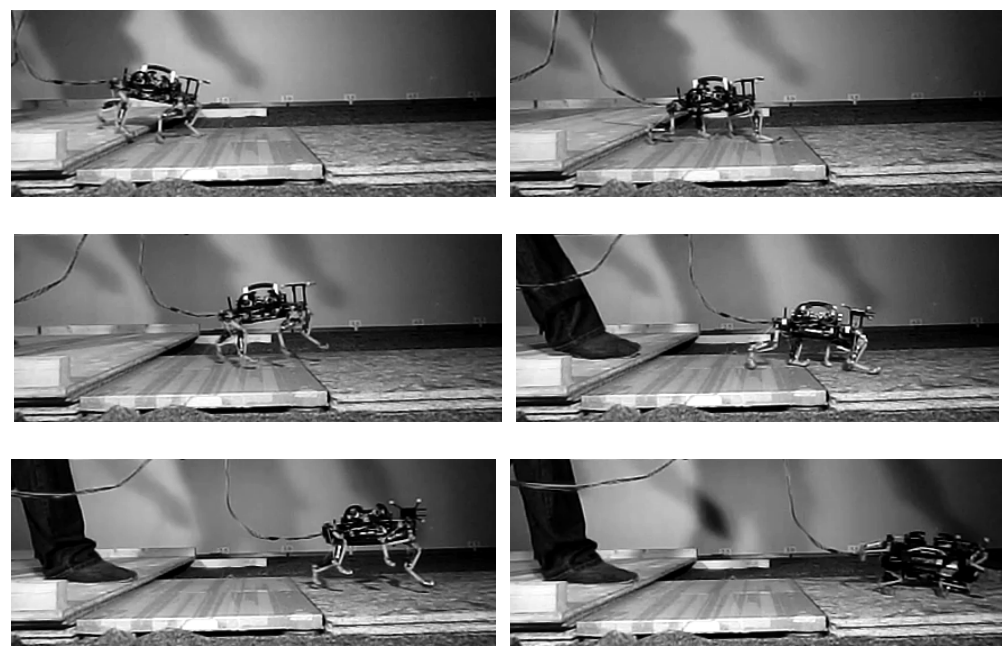

Figure 14: (Hardware experiment) Snapshot series of the robot (ASLP) failing to cross a step-down perturbation of $32 \mathrm{~mm}$. At the first and second locomotion cycle after touch-down at lower level, the robot exerted aggressive vertical jumps additionally to its forward locomotion. This eventually destabilized the robot, making it fall sideways. Time difference between pictures is $t=0.145 \mathrm{~s}$. Locomotion direction is from left to right, mean horizontal speed was $\bar{v}_{\text {hor }}=1.1 \mathrm{~m} / \mathrm{s}$. The corresponding video is shown in Extension 3 (third part).

from $0.75 \mathrm{~Hz}$ up to $3.5 \mathrm{~Hz}$.

Robot speed From Fig. 9d one can identify a mostly linear relationship between controlled stride frequency, and robot speed, for both robot configurations SLP and ASLP. The ASLP-configuration reached the maximum measured average speed, with $\bar{v}=1.42 \mathrm{~m} / \mathrm{s}$, and instantaneous peak velocities reached up to $1.65 \mathrm{~m} / \mathrm{s}$. This maximum average speed corresponds to a Froude number of 1.30. The average hip height of the running robot was $h \approx 0.125 \mathrm{~m}$. To reach robot speed values above $1 \mathrm{~m} / \mathrm{s}$, voltage was increased to gain motor speed values at the upper thermal limit. Videos for representative runs with the robot in SLP configuration, and ASLP configuration can be found in Extension 1 and Extension 2.

Hip amplitudes, and virtual duty factor Fig. 9e shows applied hip amplitudes for SLP and ASLP robot configuration runs, and Fig. 9f the corresponding virtual duty factor values. In simulation, the PSO algorithm found the parameter combination leading to the fastest gait for each stride frequency and leg configuration. At hardware experiments, parameters were iteratively tested, with earlier simulation results in mind. Hence, not only maximum amplitudes were applied. Hip amplitudes and robot speed were found to be roughly linearly related. This makes sense, since large strides can be achieved with higher hip amplitudes. Both robot configurations could apply the same range of hip amplitudes. For higher amplitudes, the SLP configuration showed more slippage on the catwalk during high-speed running (data not shown). This happened after touch-down (beginning of stance phase), and the robot slid a few millimeters 
forward every stride. Typically, this was beneficially for robot speed, but would not be directly transferable to other ground materials. On the other hand, the ASLP configuration showed very good ground contact, with almost no slippage. This indicates a more robust speed behavior. Indeed, we could easily run the ASLP robot configuration on many substrates, e.g. particle wood, parquet, table surfaces, and smooth carpeting.

Virtual duty factor $D_{\text {vir }}$ started at $0.8(\mathrm{SLP}$, at $v=0.45 \mathrm{~m} / \mathrm{s})$ up to 0.35 , for (SLP, $v=1.1 \mathrm{~m} / \mathrm{s}$ ). The highest speed gait applied a virtual duty factor of 0.43 (ASLP configuration). $D$ fluctuated from stride to stride, as the robotbody showed pitch- and roll-movements. $D$-values were typically 0.1 higher than $D_{\text {vir }}$. Fig. 15 depicts footfall patterns and individual footfall $D$-values for a representative ASLP run, at $\bar{v}=1.22 \mathrm{~m} / \mathrm{s}$.

Cost of transport Fig. 10b shows the cost of transport versus robot speed. In the lowest voltage range, maximum ASLP robot speed reached $v=0.73 \mathrm{~m} / \mathrm{s}$, at a CoT of $6.9 \mathrm{~J} / \mathrm{N} / \mathrm{m}$. The CoT minimum was reached with a ASLP configuration $(v=0.66 \mathrm{~m} / \mathrm{s}, 6.6 \mathrm{~J} / \mathrm{N} / \mathrm{m})$. In the voltage level of $U=11 \mathrm{~V}$ the SLP robot with $1.06 \mathrm{~m} / \mathrm{s}$ showed a CoT of $7.7 \mathrm{~J} / \mathrm{N} / \mathrm{m}$. The fastest gait at $13 \mathrm{~V}$ (ASLP configuration) at $1.42 \mathrm{~m} / \mathrm{s}$, applied a relatively high CoT of $9.8 \mathrm{~J} / \mathrm{N} / \mathrm{m}$. This corresponded to a total robot power consumption of $P \approx 149 \mathrm{~W}$, or an average power consumption per individual RC-servo motor of $19 \mathrm{~W}$. This power consumption is higher than the thermal limit of motors of this size, for continuous operation. Hence, high-speed experiments could only be executed with sufficient resting times. For motor voltages up to $9 \mathrm{~V}$, typically no resting times were required.

\subsubsection{Ground reaction forces, body motions}

Leg joints of the robot in ASLP configuration were tracked optically, Fig. 12 shows stick figure plots of two iterative steps, for a representative gait at $\bar{v}=$ $1.24 \mathrm{~m} / \mathrm{s}$. Plots of the first row show the flight phase of the right front leg (left plot), and the leg's stance phase (right plot). In-air leg retraction before touch-down is visible, as well as in-air leg retraction after toe-off. This is more pronounced for the front leg, less for the hind leg. Robot in-air leg retraction is qualitatively similar compared to recordings with running mammals (Alexander 1984b). In the case of the quadruped robot, neither touch-down nor toe-off in-air leg retraction was implemented, but emerged through pitch-angle dynamics of the robot. No foot slipping is visible for either front or hindlimbs. The action of the bi-articulate, second spring is visible from the non-parallel orientation of the $l_{1}$ and $l_{3}$ segment at the second half of stance phase (on the right side of stance plots), and the onsets of swing phase (left side of flight phase plots). After toe-off, the spring sets both segments back to their parallel orientation.

Ground reaction forces of a $\bar{v}=1.22 \mathrm{~m} / \mathrm{s}$ ASLP gait are provided in Fig. 15, for 2 half-steps (right force plate), and two full steps (left and right force plate). Single-leg, instantaneous vertical forces of the trotting robot reached between 0.7 body weights $(\mathrm{BW})$ and $1.1 \mathrm{BW}$. Horizontal forces reached not more than $0.3 \mathrm{BW}$, and $-0.21 \mathrm{BW}$. Qualitatively compared to GRF data of (heavier) trotting dogs (Lee et al. 1999, Fig. 2, p. 3567) the shown here GRF profiles are less symmetric (vertical component), and fluctuate more.

In Fig. 16, GRF are plotted combined into a single 2D-vector per leg (iden- 


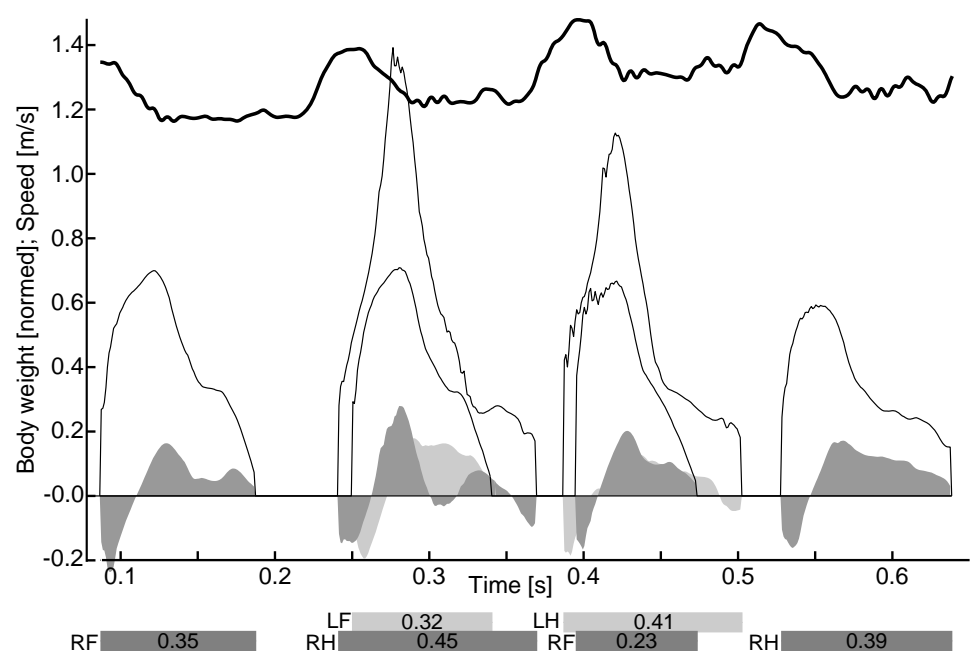

Figure 15: (Hardware experiment) Plotted are ground reaction forces (GRF) from two side-by-side mounted force plates (FP, left and right), center of mass speed (COM-speed), and footfall patterns of a typical experiment with the robot, leg configuration ASLP. The robot ran with its left legs over the left FP, and with its right legs over the right FP. COM-speed showed an average of $1.22 \mathrm{~m} / \mathrm{s}$, peak speed was around $1.48 \mathrm{~m} / \mathrm{s}$. Vertical forces (thin lines) and foreaft forces (gray areas) are plotted in multiples of body-weight (BW, $m=1.1 \mathrm{~kg}$ ). Side-forces are omitted for clarity but existed because of body roll-motions during locomotion. COM-speed (thick line) is plotted in $\mathrm{m} / \mathrm{s}$. Footfall patterns are provided, numerical values indicate individual leg duty factor values. Six footfall GRF patterns are shown, the robot stepped with a single (right front) leg onto the right force plate, and it left the force plate area with its right rear leg. Therefore, only for the second and third stride the sum of vertical forces of two diagonal legs added up to roughly one body weight per stride. Net speed of the robot is increasing slightly during the presented time window in strides two and three. These stride-to-stride fluctuations of speed showed up frequently: actuator energy insertion was fixed by the gait pattern, but local changes in ground conditions (friction, rough ground) led to small changes in robot speed. Fore-aft forces are equally indicating increasing speed: positive net fore-aft force values are measured in this example (second and third stride $0.08 \mathrm{BW}$ ).

tical data as in Fig. 15), and the robot's center of mass position over time. The combined vertical and horizontal GRF point inwards, towards the robot's center of mass, for snapshots of the first half of the stance phase. At the late stance phase, the combined hind leg GRF points more outwards (Fig. 16d-f). Inwards pointing GRF are associated to a higher dynamical self-stability against robot pitching motions (Lee et al. 2005; Maus et al. 2010).

\subsubsection{Step-down experiments}

To document gait robustness further than for running on level ground, we conducted step-down experiments with three step-down heights, and for both leg configurations SLP and ASLP. A step of $32 \mathrm{~mm}$ presents $20 \%$ of the robot's 


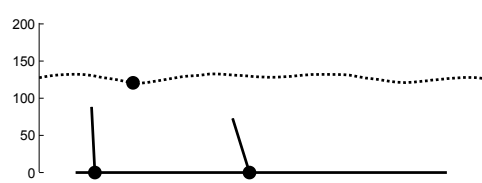

(a) $0.000 \mathrm{~s}$

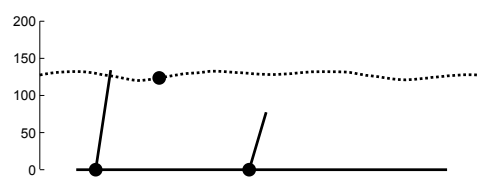

(c) $0.030 \mathrm{~s}$

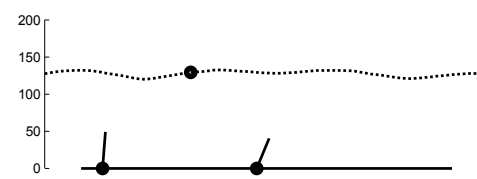

(e) $0.060 \mathrm{~s}$

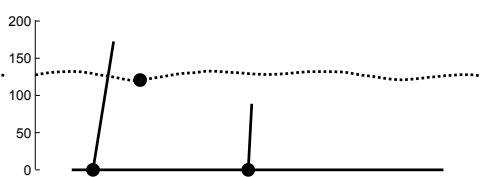

(b) $0.015 \mathrm{~s}$

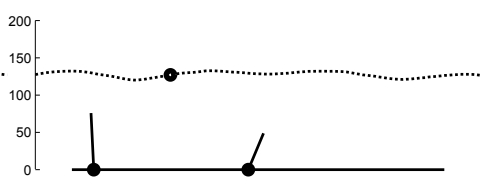

(d) $0.045 \mathrm{~s}$

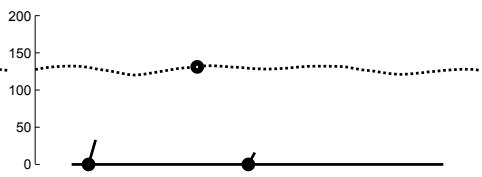

(f) $0.075 \mathrm{~s}$

Figure 16: (Hardware experiment) Planar presentation of ground reaction force vector of two diagonal limbs (trot gait) and the robot's center of mass (COM) during a single stance phase. The robot (ASLP configuration) ran from left to right, at $f=3.5 \mathrm{~s}^{-1}$, with a speed of $\bar{v}=1.22 \mathrm{~m} / \mathrm{s}$. Left force vector presents right limb GRF (right hind leg), right force vector left limb GRF (left front leg). The dotted line is tracking the COM position over time. The horizontal bold line represents the force plate (length $l=0.5 \mathrm{~m}$ ). Vertical scale indicates height of $\mathrm{COM}$ in $[\mathrm{mm}]$.

standing hip height (Table 2), and a touch-down (TD) delay of more than 20 msec. Typical stance times on level ground ranged between $70 \mathrm{msec}$ and $110 \mathrm{msec}$, for the applied gait. CPG parameters for both configurations were adapted, because a direct parameter transfer from SLP to ASLP was not successfully reproducing a stable gait. Both configurations ran between $\bar{v}=1.15 \mathrm{~m} / \mathrm{s}$ (SLP configuration) and $\bar{v}=1.18 \mathrm{~m} / \mathrm{s}$ (ASLP configuration). 20 step-down experiments per configuration and step-down height were performed, for stepdown heights of $12 \mathrm{~mm}, 20 \mathrm{~mm}$, and $32 \mathrm{~mm}$ (120 experiments in total). Gaits were marked as successful if the robot would run until the end of the catwalk, about $1.5 \mathrm{~m}$ after the step-down, and showed no further signs of instability. Fig. 17 gives the number of successful step-downs, videos of successful and unsuccessful ASLP step-down experiments can be found in Extension 3. For all step-down heights, the ASLP configuration performed better. For the biggest step-down $(h=32 \mathrm{~mm}), 20 \%$ of runs with the ASLP configuration were successful, and $10 \%$ of runs with the SLP configuration.

\subsection{3 $\quad A_{\mathrm{st}}^{\mathrm{k}}$ drive function}

This section builds on the hypothesis that a certain $A_{\mathrm{st}}^{\mathrm{k}}$ control drive is necessary to support robust speed transition and control within trot gait. We gained first indicators of the importance of $A_{\mathrm{st}}^{\mathrm{k}}$ control through results with the simulated 


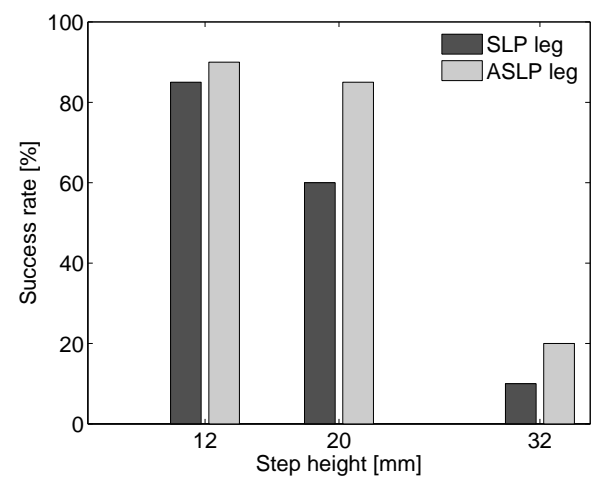

Figure 17: (Hardware experiment) Results from step-down experiments. 20 experiments per leg configuration per step-down height were executed, results from 120 runs are shown. The robot passed the step-down with success in case it did not fall directly at the step-down, and did not show signs of instability until it reached the end of the catwalk. Success rates are plotted in \%. A $32 \mathrm{~mm}$ step-down correspond to $20 \%$ of the standing leg length. The robot-gait was a running trot, forward speed between $1.1 \mathrm{~m} / \mathrm{s}$ and $1.2 \mathrm{~m} / \mathrm{s}$.

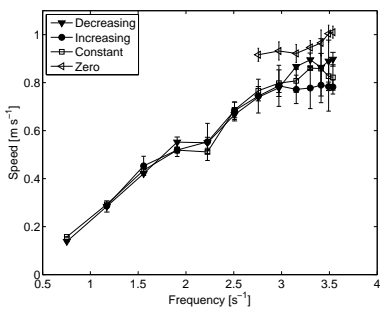

(a) Robot speed

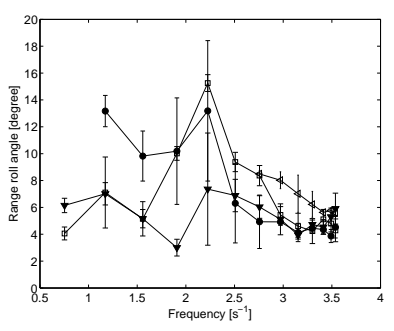

(b) Pitch angle

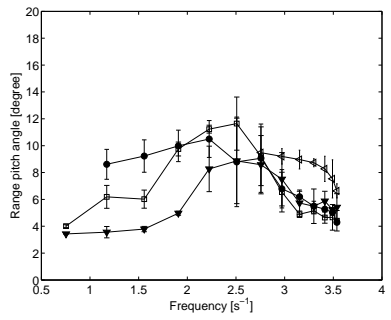

(c) Roll angle

Figure 18: (Hardware experiment) Speed, pitch-angle, and roll-angle versus stride frequency, for four different $A_{\mathrm{st}}^{\mathrm{k}}$-strategies. Symbols are as in Fig. 18a. Only runs above a frequency of $2.5 \mathrm{~Hz}$ and a speed of $0.9 \mathrm{~m} / \mathrm{s}$ could be run with passive stance leg length strategy ( $A_{\mathrm{st}}^{\mathrm{k}}$-zero strategy, empty triangle symbols). $A_{\mathrm{st}}^{\mathrm{k}}$-decreasing showed the lowest pitch- and roll-angles. The $A_{\mathrm{st}}^{\mathrm{k}}$-zero control resulted in the fastest gaits, within the applied frequency range. With no active stance phase deflection the leg was longer during stance phase. This eventually resulted in larger stride lengths, and higher pitch- and roll-angles. RC servo motor voltage was kept constant in this experiment, at $10.5 \mathrm{~V}$.

Cheetah-cub (Section 4.1). The optimization algorithm found gaits with the simulated robot, where $A_{\mathrm{st}}^{\mathrm{k}}$ was lowered mostly linearly between low-speed and high-speed (Fig. 11). We hypothesized that increasing the forward robot speed increases momentum-based leg spring deflection. The faster the robot is, the more its spring-loaded legs are being compressed by robot-momentum while in stance phase. This simultaneously shortens leg length, and self-stabilizes trot gait locomotion. On the contrary, with little forward speed, the stance-leg springs will not be compressed through body momentum. For low robot speed, $A_{\mathrm{st}}^{\mathrm{k}}$ takes over the function of compressing legs during mid-stance, and reduces 
vertical hip movements of the robot. Leg length actuation is implemented on Cheetah-cub with a cable mechanism. The cable force will either compress the leg during mid-stance (Fig. 8), or in case body momentum is higher, the cable will go slack and body momentum will compress the stance-leg. The proposed hypothesis has one further conclusion: at a certain robot speed, no actuated $A_{\mathrm{st}}^{\mathrm{k}}$ control would be necessary anymore. Rather, the stance-leg length will be compressed sufficiently by body momentum, this will self-stabilize the trotting robot.

We tested the above hypothesis on the robot by implementing four types of $A_{\mathrm{st}}^{\mathrm{k}}$ drive functions: decreasing, constant, increasing, and zero- $A_{\mathrm{st}}^{\mathrm{k}}$ actuation over speed (Table 6, four last rows). As a testing platform, we chose the ASLP configuration, as it performed more robustly in experiments before (Fig. 17). The other CPG parameters were not kept constant but also transferred into a single drive function (Table 6) that takes the desired speed as a driving parameter. The shape of these functions was extracted from earlier experiments (Fig. 9d-9f). Fig. 18 depicts the results from three repeated experiments per $A_{\mathrm{st}}^{\mathrm{k}}$ drive-function strategy, and per frequency.

Table 6: CPG parameter drive functions, for ASLP robot configuration, derived from experiments in Fig. 9. Row 9-12 documents drive functions tested for four $A_{\mathrm{st}}^{\mathrm{k}}$ strategies (Section 4.2.3). Here, $v$ is the desired forward speed.

\begin{tabular}{rlr} 
& Param. & Function \\
\hline 1 & $A^{\mathrm{h}}$ & $12 * v^{3}-9.3 * v^{2}+48 * v+19$ \\
2 & $f$ & $-1.7 * v^{2}+4.7 * v+0.3$ \\
3 & $D$ & $-0.18 * v+0.66$ \\
4 & $O_{\mathrm{fr}}^{\mathrm{h}}$ & $1.8 * v^{3}-6.8 * v^{2}+5 * v+14$ \\
5 & $O_{\mathrm{fr}}^{\mathrm{k}}$ & $-0.14 * v^{2}+0.12 * v+0.38$ \\
6 & $O_{\mathrm{fr}}^{\mathrm{h}}$ & $4.4 * v^{3}-17 * v^{2}+12 * v+13$ \\
7 & $A_{\mathrm{sw}}^{\mathrm{k}}$ & $-0.44 * v^{3}+1.7 * v^{2}-1.2 * v+0.71$ \\
8 & $O_{\mathrm{hi}}^{\mathrm{k}}$ & $-0.28 * v^{2}+0.24 * v+0.16$ \\
9 & $A_{\mathrm{st}}^{\mathrm{k}}$ dec. & $-0.21 * v+0.32$ \\
10 & $A_{\mathrm{st}}^{\mathrm{k}}$ inc. & $0.21 * v+0.03$ \\
11 & $A_{\mathrm{st}}^{\mathrm{k}}$ con. & 0.15 \\
12 & $A_{\mathrm{st}}^{\mathrm{k}}$ zero & 0
\end{tabular}

Fig. 18a shows a mostly linear robot speed response for the four tested $A_{\mathrm{st}}^{\mathrm{k}}$-strategies. For $A_{\mathrm{st}}^{\mathrm{k}}$-increasing, no stable gait could be found at $0.75 \mathrm{~Hz}$. For the $A_{\mathrm{st}}^{\mathrm{k}}$-zero strategy, only frequencies above $2.75 \mathrm{~Hz}$ produced valid gaits. These $A_{\mathrm{st}}^{\mathrm{k}}$-zero gaits were $\approx 0.1 \mathrm{~m} / \mathrm{s}$ faster than gaits of the remaining three $A_{\mathrm{st}}^{\mathrm{k}}$-strategies (inc, dec, con). Fig. 18b and Fig. 18c indicate pitch- and rollangles of the body. $A_{\mathrm{st}}^{\mathrm{k}}$-decreasing shows in average the lowest roll- and pitchangle movements over all frequencies. $A_{\mathrm{st}}^{\mathrm{k}}$-inc produced valid gaits, but also more than double as high pitch-angle movements compared to $A_{\mathrm{st}}^{\mathrm{k}}$-decreasing. Roll- and pitch-angles of $A_{\mathrm{st}}^{\mathrm{k}}$-zero decreased with increasing robot speed (from $v=0.9 \mathrm{~m} / \mathrm{s}$ on) and stride frequencies. This indicates that with the $A_{\mathrm{st}}^{\mathrm{k}}$-zero strategy, the robot had sufficient body momentum to self-compress its stance legs. The low roll- and pitch-angles of the $A_{\mathrm{st}}^{\mathrm{k}}$-decreasing strategy suggest that active $A_{\mathrm{st}}^{\mathrm{k}}$ control worked best up to a speed of $\approx 1 \mathrm{~m} / \mathrm{s}$, for the chosen maximum motor coil voltage. 


\section{Discussion}

The main outcome of this work is the description and documentation of an open loop, self-stabilizing, four-segment, compliant quadruped robot trotting dynamically on flat ground. The robot could reach speeds up to $\bar{v}=1.42 \mathrm{~m} / \mathrm{s}$ (ASLP-configuration). The robot successfully overcame small step-down perturbations, up to $20 \%$ of its standing hip height. The SLP-configuration was measurably less robust in step-down perturbation experiments for all tested step-down heights, compared to the ASLP-leg design. An important parameter for robust, open loop trotting was the active reduction of leg length during midstance (active knee stance deflection, $A_{\mathrm{st}}^{\mathrm{k}}$ ). It allowed the robot to trot through a large range of speeds, with a minimum of roll- and pitch-movements.

The subject of our first hypothesis was an expected higher cost of transport (CoT) for the SLP leg configuration, compared to the ASLP leg configuration. By adding in-series elastic elements distally, we expected a lower power consumption at the same speed, according to observations in biology (Alexander 1984a). While we observed in average a CoT reduction in simulation (Section 4.1, Fig. 10a), this difference was not found in the hardware experiments (Fig. 10b). CoT in simulation ranged between $0.25 \mathrm{~J} / \mathrm{N} / \mathrm{m}$, up to $1.25 \mathrm{~J} / \mathrm{N} / \mathrm{m}$. CoT of hardware runs was factor 15 higher. The smallest CoT measured was $6.6 \mathrm{~J} / \mathrm{N} / \mathrm{m}$, with the ASLP configuration. A major difference between simulation and hardware experiments was the actuator modeling (Webots) and implementation (hardware robot). RC servo motors were modeled in Webots with their corresponding maximum speed and stall torque values. However, the applied $\mathrm{RC}$ servo motors had also attached a $300: 1$ gearbox with 5 spur gear pairs. Due to the complexity of motor/gearbox modeling, RC servo motor losses were not included in the simulation. Instead, actuators were modeled as loss-free, PIDcontrolled torque sources. Roos et al. (2006) show that main actuator losses for high-speed (high frequency, and direction changes) and high-load applications root in reflected inertia effects of gearbox and motor coil. Due to the RC servo motors' high gearbox ratio, these higher losses by a factor 15 are plausible. High motor losses were also recognizable by high motor temperatures at fast robot runs. These losses can potentially be avoided by applying a design with an optimized motor/gearbox combination.

In our second hypothesis we established a connection between robot speed, robot-stability, and active leg length shortening during stance phase for the spring-loaded, compliant robot leg design. Indeed, we could show that with an $A_{\mathrm{st}}^{\mathrm{k}}$-dec strategy the robot was stable in the largest range of speed. It was selfstabilizing with increasing robot speed, and it showed the lowest roll- and pitchangle body movements. The $A_{\mathrm{st}}^{\mathrm{k}}$-zero strategy could also run the robot, but only at the highest robot speed range. Hence, at higher robot speed, robot forward momentum was gradually taking over the function of compressing the leg spring during stance phase. At lower robot speeds, active leg length shortening of the spring-loaded leg successfully decreased destabilizing roll-angle and pitch-angle movements.

Cheetah-cub outperformed other quadruped legged robots, in trot gait, in terms of Froude number (Fig. 3). However, Cheetah-cub's speed comes at the cost of running a relatively bad energy economy. For example, the $22 \mathrm{~kg}$ Scout II robot (Smith et al. 2004) reported a specific electrical resistance of 1.7 at $1.3 \mathrm{~m} / \mathrm{s}$. Cheetah-cub shows a CoT of $10 \mathrm{~J} / \mathrm{N} / \mathrm{m}(149 \mathrm{~W})$ at roughly the same 
speed, at $f=3.5 \mathrm{~Hz}$ locomotion frequency. Kuo (2007) provides a list of CoTvalues of animals and robots, Cheetah-cub must be placed at the upper bound of its field.

Cheetah-cub is currently speed optimized, and no actuators for leg adduction and abduction are included. Such actuators would facilitate steering, but increase robot-weight. For convenient experimentation (no battery loading or exchange), Cheetah-cub was powered in all experiments externally by a tether.

A novelty in dynamic robot-trotting is the self-stabilizing property of the robot (Fig. 18). Therefore, the robot can be controlled with simple, speed dependent, open loop patterns. Other legged, quadruped robot-platforms typically require feedback on flat terrain, their performance is measured with feedback signals in the loop. The proposed robot design robustly runs open loop on flat terrain and with step-down perturbations. Hence, here we described the mechanical and open loop robot characteristics of the system. We expect that the separation between open loop stability, and future closed-loop control will allow us to better characterize the effects of potential feedback strategies.

\section{Conclusion}

We presented a novel quadruped robot design which enables fast and dynamic quadruped trot gaits over a large range of speeds. The robot reached a maximum speed of $\bar{v}=1.42 \mathrm{~m} / \mathrm{s}$, corresponding to a Froude number of 1.3 . This is currently the highest Froude number reported for a trotting quadruped, to the best of our knowledge. We proposed a robot design which is small and lightweight $(m=1.1 \mathrm{~kg})$, compared to large quadruped robots like BigDog (Raibert et al. 2008). This allowed us to conveniently perform experiments within our small laboratory. Because of its size and design, the robot is cheap and easy to replicate, repair, and extend. The derived robot design and its open loop control exhibited excellent self-stability, in a large range of speeds and in the presence of step-down perturbations. The two tested leg configurations (spring loaded pantograph: SLP, and advanced spring loaded pantograph: ASLP) were not too different in terms of speed and cost of transport, on the hardware platform. We could however provide results from hardware step-down experiments, where ASLP performed more robustly, due to its distally placed, in-series elastic elements. ASLP performed also more robustly on different grounds. It was sliding less than the leg design without a compliant foot (SLP). For leg length control, we found that a speed-dependent adaptation of the mid-stance leg length ( $A_{\mathrm{st}}^{\mathrm{k}}$ parameter) greatly increased robustness, for the suggested ASLP leg design. In the future, we will tackle hardware design topics like a fully tether-less robot setup, leg force-sensing, and steering. For trotting in rough terrain, the platform will require feedback controllers, and adequate sensor designs.

\section{Appendix A: Index to Multimedia Extensions}

The multimedia extensions to this article are at:

http: //www.ijrr.org 


\begin{tabular}{ccl}
\hline Extension & Type & Description \\
\hline 1 & Video & Hallway run \\
2 & Video & Trot gait with SLP legs $(\bar{v}=1.15 \mathrm{~m} / \mathrm{s})$ \\
& & Trot gait with ASLP legs $(\bar{v}=1.25 \mathrm{~m} / \mathrm{s})$ \\
& & Webots simulation - Trot gait $\left(f=3 \mathrm{~s}^{-1}\right)$ \\
3 & Video & Successful step-down experiment $(20 \%$ leg length $)$ \\
& & Successful step-down experiment $(12 \%$ leg length $)$ \\
& & Unsuccessful step-down experiment (20\% leg length) \\
\hline
\end{tabular}

\section{Acknowledgment}

We thank Mahdi Khoramshahi and Rolando Rodas for assisting with experiments and robot assembly. We thank Jesse van den Kieboom, who developed code, and provided infrastructure for the PSO-based optimization framework. The research leading to these results has received funding from the European Community's Seventh Framework Programme FP7/2007-2013-Challenge 2-Cognitive Systems, Interaction, Robotics-under grant agreement No. 248311 (AMARSi), and from the Swiss National Science Foundation through the National Centre of Competence in Research Robotics.

\section{References}

Alexander, R. (Jan. 1984a). "Elastic Energy Stores in Running Vertebrates". In: American Zoologist 24.1, pp. $85-94$.

- (1984b). "The Gaits of Bipedal and Quadrupedal Animals". In: The International Journal of Robotics Research 3.2, pp. 49-59.

- (1989). "Optimization and gaits in the locomotion of vertebrates". In: Physiological reviews 69.4, 1199-1227 (106 ref.)

- (1996). "Walking and Running". In: The Mathematical Gazette 80.488, pp. 262266.

Altendorfer, R., N. Moore, H. Komsuoglu, M. Buehler, H. Brown, D. McMordie, U. Saranli, R. Full, and D. Koditschek (Nov. 2001). "RHex: A Biologically Inspired Hexapod Runner". In: Autonomous Robots 11.3, pp. 207-213.

Barre, A. and S. Armand (2011). b-tk Biomechanical ToolKit.

Battaglia, R. (1999). Design of the SCOUT II quadruped with preliminary stairclimbing. Master thesis. McGill University, Canada.

Biewener, A. A (1983). "Allometry of Quadrupedal Locomotion: The Scaling of Duty Factor, Bone Curvature and Limb Orientation to Body Size". In: Journal of Experimental Biology 105.1, pp. 147-171.

Blickhan, R. (1989). "The spring-mass model for running and hopping". In: Journal of Biomechanics 22.11-12, pp. 1217-1227.

Brown, D. (2012). Video analysis and modeling tool.

Buehler, M., R. Battaglia, A. Cocosco, G. Hawker, J. Sarkis, and K. Yamazaki (1998). "SCOUT: a simple quadruped that walks, climbs, and runs". In: Robotics and Automation, 1998. Proceedings. 1998 IEEE International Conference on. Vol. 2, 1707-1712 vol.2. 
Buehler, M., R. Playter, and M. Raibert (2005). "Robots step outside". In: AMAM2005.

Cavagna, G. A., N. C. Heglund, and C. R. Taylor (Nov. 1977). "Mechanical work in terrestrial locomotion: two basic mechanisms for minimizing energy expenditure". In: Am J Physiol Regul Integr Comp Physiol 233.5, R243-261.

Daley, M. A. and A. A. Biewener (Oct. 2006). "Running over rough terrain reveals limb control for intrinsic stability". In: Proceedings of the National Academy of Sciences 103.42, pp. 15681-15686.

Degallier Rochat, S., L. Righetti, S. Gay, and A. Ijspeert (2011). "Towards simple control for complex, autonomous robotic applications: Combining discrete and rhythmic motor primitives". In: Autonomous Robots 31.2, pp. 155181.

Eberhart, R. and J. Kennedy (Oct. 1995). "A new optimizer using particle swarm theory". In: , Proceedings of the Sixth International Symposium on Micro Machine and Human Science, 1995. MHS '95. IEEE, pp. 39-43.

Estremera, J. and K. J. Waldron (2008). "Thrust Control, Stabilization and Energetics of a Quadruped Running Robot". In: The International Journal of Robotics Research 27.10, pp. 1135-1151.

Fischer, M. and R. Blickhan (2006). "The tri-segmented limbs of therian mammals: kinematics, dynamics, and self-stabilization-a review". In: Journal of Experimental Zoology. Part A, Comparative Experimental Biology 305.11, pp. 935-952.

Fukuoka, Y. and H. Kimura (2009). "Dynamic locomotion of a biomorphic quadruped Tekken-robot using various gaits: walk, trot, free-gait and bound". In: Applied Bionics and Biomechanics 6.1, pp. 63-71.

Fukuoka, Y., H. Kimura, and A. H. Cohen (2003). "Adaptive Dynamic Walking of a Quadruped Robot on Irregular Terrain Based on Biological Concepts". In: The International Journal of Robotics Research 22.3-4, pp. 187 -202.

Full, R. and D. Koditschek (Dec. 1999). "Templates and anchors: neuromechanical hypotheses of legged locomotion on land". In: Journal of Experimental Biology 202.23, pp. $3325-3332$.

Gabrielli, G. and T. von Karman (1950). "What price speed?: specific power required for propulsion of vehicles". In: Mechanical Engineering, pp. 775781.

Geyer, H., A. Seyfarth, and R. Blickhan (Nov. 2006). "Compliant leg behaviour explains basic dynamics of walking and running". In: Proceedings of the Royal Society B: Biological Sciences 273.1603, pp. 2861-2867.

Gregersen, C. S., N. A. Silverton, and D. R. Carrier (Dec. 1998). "External work and potential for elastic storage at the limb joints of running dogs". In: The Journal of Experimental Biology 201.Pt 23. PMID: 9808833, pp. 3197-3210.

Halbertsma, J. (1983). "The stride cycle of the cat: the modelling of locomotion by computerized analysis of automatic recordings." In: Acta Physiologica Scandinavica, Supplement 521, pp. 1-75.

Hawker, G. and M. Buehler (2000). "Quadruped trotting with passive knees: design, control, and experiments". In: IEEE International Conference on Robotics and Automation, 2000. Proceedings. ICRA '00. Vol. 3. IEEE, 30463051 vol.3.

Herr, H. M. and T. A. McMahon (June 2000). "A Trotting Horse Model". In: The International Journal of Robotics Research 19.6, pp. 566-581. 
Hutter, M., C. Gehring, M. Bloesch, M. Hoepflinger, C. Remy, and R. Siegwart (2012). "StarlETH: A compliant quadrupedal robot for fast, efficient, and versatile locomotion". In: Int. Conf. on Climbing and Walking Robots.

Iida, F., G. Gomez, and R. Pfeifer (2005). "Exploiting body dynamics for controlling a running quadruped robot". In: , 12th International Conference on Advanced Robotics, 2005. ICAR '05. Proceedings. IEEE, pp. 229-235.

Iida, F. and R. Pfeifer (2004). "Cheap rapid locomotion of a quadruped robot: Self-stabilization of bounding gait". In: Intelligent Autonomous Systems.

Ijspeert, A. J. (2008). "Central pattern generators for locomotion control in animals and robots: a review". In: Neural Networks 21.4, pp. 642-653.

Ijspeert, A. J., A. Crespi, D. Ryczko, and J. Cabelguen (Mar. 2007). "From Swimming to Walking with a Salamander Robot Driven by a Spinal Cord Model". In: Science 315.5817, pp. 1416-1420.

Kim, S., J. E. Clark, and M. R. Cutkosky (2006). "iSprawl: Design and Tuning for High-speed Autonomous Open-loop Running". In: The International Journal of Robotics Research 25.9, pp. 903-912.

Kimura, H., Y. Fukuoka, and A. Cohen (2007). "Adaptive Dynamic Walking of a Quadruped Robot on Natural Ground Based on Biological Concepts". In: The International Journal of Robotics Research 26.5, pp. 475-490.

Kistler (2011). measure, analyze, innovate. Winterthur, Switzerland: Kistler Instrumente AG.

Kohl, N. and P. Stone (2004). "Policy gradient reinforcement learning for fast quadrupedal locomotion". In: 2004 IEEE International Conference on Robotics and Automation, 2004. Proceedings. ICRA '04. Vol. 3. IEEE, 2619-2624 Vol.3.

Kuo, A. (2007). "Choosing Your Steps Carefully". In: Robotics 83 Automation Magazine, IEEE 14.2, pp. 18-29.

Lee, D., J. Bertram, and R. Todhunter (Dec. 1999). "Acceleration and balance in trotting dogs". In: J Exp Biol 202.24, pp. 3565-3573.

Lee, D. and S. G. Meek (Mar. 2005). "Directionally compliant legs influence the intrinsic pitch behaviour of a trotting quadruped". In: Proceedings of the Royal Society B: Biological Sciences 272.1563, pp. 567-572.

MATLAB (2009). version 7.09.0 (R2009b). Natick, Massachusetts: The MathWorks Inc.

Maus, H., S. Lipfert, M. Gross, J. Rummel, and A. Seyfarth (Sept. 2010). "Upright human gait did not provide a major mechanical challenge for our ancestors". In: Nature Communications 1, p. 70.

More, H. L., J. R. Hutchinson, D. F. Collins, D. J. Weber, S. K. H. Aung, and J. M. Donelan (July 2010). "Scaling of Sensorimotor Control in Terrestrial Mammals". In: Proceedings of the Royal Society B: Biological Sciences 277.1700 , pp. 3563-3568.

Narioka, K. and K. Hosoda (May 2011). "Motor development of an pneumatic musculoskeletal infant robot". In: 2011 IEEE International Conference on Robotics and Automation (ICRA), pp. 963 -968.

Naturalpoint, Inc. (2011). Optitrack s250e. Corvallis, Oregon.

Poulakakis, I., J. A. Smith, and M. Buehler (2005). "Modeling and Experiments of Untethered Quadrupedal Running with a Bounding Gait: The Scout II Robot". In: The International Journal of Robotics Research 24.4, pp. 239256. 
Poulakakis, I., E. Papadopoulos, and M. Buehler (July 2006). "On the Stability of the Passive Dynamics of Quadrupedal Running with a Bounding Gait". In: The International Journal of Robotics Research 25.7, pp. 669-687.

Raibert, M., M. Chepponis, and H. Brown (1986). "Running on four legs as though they were one". In: Robotics and Automation, IEEE Journal of 2.2, pp. $70-82$.

Raibert, M., K. Blankespoor, G. Nelson, and R. Playter (2008). "BigDog, the Rough-Terrain Quadruped Robot". In: Proceedings of the 17th IFAC World Congress. COEX, South Korea, pp. 10823-10825.

Raibert, M. (1990). "Trotting, pacing and bounding by a quadruped robot". In: Journal of Biomechanics 23.Supplement 1, pp. 79-81.

Righetti, L. and A. J. Ijspeert (2008). "Pattern generators with sensory feedback for the control of quadruped locomotion". In: Proceedings of the 2008 IEEE International Conference on Robotics and Automation (ICRA 2008). Pasadena, pp. 819-824.

Righetti, L., J. Buchli, and A. J. Ijspeert (2009). "Adaptive Frequency Oscillators and Applications". In: The Open Cybernetics and Systemics Journal 3, pp. 64-69.

Roos, F., H. Johansson, and J. Wikander (Feb. 2006). "Optimal selection of motor and gearhead in mechatronic applications". In: Mechatronics 16.1, pp. 63-72.

Rutishauser, S., A. Spröwitz, L. Righetti, and A. J. Ijspeert (2008). "Passive compliant quadruped robot using central pattern generators for locomotion control". In: 2008 IEEE International Conference on Biomedical Robotics and Biomechatronics. Scottsdale.

Sato, T., T. Kano, and A. Ishiguro (June 2011). "On the applicability of the decentralized control mechanism extracted from the true slime mold: a robotic case study with a serpentine robot". In: Bioinspiration $\mathcal{E}$ Biomimetics 6.2, p. 026006.

Schmidt, M. and M. Fischer (2009). "Morphological Integration in Mammalian Limb Proportions: Dissociation between Function and Development". In: Evolution 63.3, pp. 749-766.

Schroer, R. T., M. J. Boggess, R. J. Bachmann, R. D. Quinn, and R. E. Ritzmann (2004). "Comparing cockroach and whegs robot body motion". In: in: proceedings of the ieee international conference on robotics and automation, april 2004, pp. 3288-3293.

Semini, C., N. G. Tsagarakis, E. Guglielmino, M. Focchi, F. Cannella, and D. G. Caldwell (2011). "Design of HyQ - a hydraulically and electrically actuated quadruped robot". In: IMechE, Part I: Journal of Systems and Control Engineering 225.6, pp. $831-849$.

Seyfarth, A., H. Geyer, M. Guenther, and R. Blickhan (2002). "A movement criterion for running". In: Journal of Biomechanics 35.5, pp. 649-655.

Smith, J. A and I. Poulakakis (2004). "Rotary gallop in the untethered quadrupedal robot scout II". In: 2004 IEEE/RSJ International Conference on Intelligent Robots and Systems, 2004. (IROS 2004). Proceedings. Vol. 3. IEEE, pp. 2556-2561.

Smith, J. A., I. Poulakakis, M. Trentini, and I. Sharf (2010). "Bounding with Active Wheels and Liftoff Angle Velocity Adjustment". In: The International Journal of Robotics Research 29.4, pp. $414-427$. 
Spröwitz, A., R. Moeckel, J. Maye, and A. J. Ijspeert (Mar. 2008). "Learning to Move in Modular Robots using Central Pattern Generators and Online Optimization". In: The International Journal of Robotics Research 27.3-4, pp. 423-443.

Taga, G. (1994). "Emergence of bipedal locomotion through entrainment among the neuro-musculo-skeletal system and the environment". In: Physica D: Nonlinear Phenomena 75.1-3, pp. 190-208.

Tucker, V. A. (June 1970). "Energetic cost of locomotion in animals". In: Comparative Biochemistry and Physiology 34.4, pp. 841-846.

Umedachi, T., K. Takeda, T. Nakagaki, R. Kobayashi, and A. Ishiguro (2010). "Fully decentralized control of a soft-bodied robot inspired by true slime mold". In: Biological Cybernetics 102.3, pp. 261-269.

Watanabe, W., T. Sato, and A. Ishiguro (2009). "A fully decentralized control of a serpentine robot based on the discrepancy between body, brain and environment". In: Intelligent Robots and Systems, 2009. IROS 2009. IEEE/RSJ International Conference on, pp. 2421-2426.

Webots (2009). http://www.cyberbotics.com. Ed. by C. Ltd. Commercial Mobile Robot Simulation Software.

Witte, H., R. Hackert, K. Lilje, N. Schilling, D. Voges, G. Klauer, W. Ilg, J. Albiez, A. Seyfarth, D. Germann, M. Hiller, R. Dillmann, and M. Fischer (2001). "Transfer of biological principles into the construction of quadruped walking machines". In: Robot Motion and Control, 2001 Proceedings of the Second International Workshop on, pp. $245-249$.

Witte, H., R. Hackert, W. Ilg, J. Biltzinger, N. Schillinger, F. Biedermann, M. Jergas, H. Preuschoft, and M. Fischer (2003). "Quadrupedal Mammals as Paragons for Walking Machines". In: Proc AMAM - Adaptive Motion in Animals and Machines, TuA-II-2.1 -TuA-II-2.4.

Witte, H., J. Biltzinger, R. Hackert, N. Schilling, M. Schmidt, C. Reich, and M. Fischer (2002). "Torque patterns of the limbs of small therian mammals during locomotion on flat ground". In: J Exp Biol 205, pp. 1339-1353.

Zeglin, G. (1999). "The Bow Leg Hopping Robot". PhD thesis. CMU, USA.

Zhang, Z. G. and H. Kimura (2009). "Rush: a simple and autonomous quadruped running robot". In: Proceedings of the Institution of Mechanical Engineers, Part I: Journal of Systems and Control Engineering 223.3, pp. 323-336. 